\title{
Periodic orbits near bifurcations of codimension two: Classical mechanics, semiclassics and Stokes transitions
}

\author{
Henning Schomerus \\ Fachbereich Physik, Universität-Gesamthochschule Essen, D-45117 Essen, Germany
}

Received 12 November 1997, in final form 20 February 1998

\begin{abstract}
We investigate classical and semiclassical aspects of codimension-two bifurcations of periodic orbits in Hamiltonian systems. A classification of these bifurcations in autonomous systems with two degrees of freedom or time-periodic systems with one degree of freedom is presented. We derive uniform approximations to be used in semiclassical trace formulae and determine also certain global bifurcations in conjunction with Stokes transitions that become important in the ensuing diffraction catastrophe integrals.
\end{abstract}

\section{Introduction}

Periodic-orbit theory aims at the semiclassical evaluation of energy levels of quantum systems and relates their spectral properties to periodic orbits of the corresponding classical system. For autonomous systems one considers the trace of the Green function $G(E)$ which determines also the density of states $d(E)$. For periodically driven systems the objects of interest are the traces $\operatorname{tr} F^{n}$ of the stroboscopic time-evolution operator over $n$ periods; they encode the so-called quasienergies of states that are stationary in the stroboscopic description. Both types of traces can be written as a sum of individual contributions of periodic orbits for chaotic (hyperbolic) systems [1-4] or a sum over rational tori for integrable motion $[5,6]$.

Recent semiclassical studies [7-12] based on periodic-orbit theory were devoted to the neighbourhood, in the space of control parameters, of classical bifurcations. These are instances in which periodic orbits coalesce and are the mechanism how orbits are born or disappear, or change their configuration when the energy or an external parameter is varied. Bifurcations are ubiquitous in systems with a mixed phase space and pave the path from integrable to chaotic motion.

A collective treatment of the bifurcating orbits was found necessary, and even more the inclusion of predecessors of such orbits which live in complexified phase space and were called ghosts. A collective contribution comes from an orbit cluster; only when far away from the bifurcation can it be written as a sum of individual contributions. Both types of contributions are an additive term in the periodic-orbit expansion of the trace in question.

The existing semiclassical (and most of the classical) studies focus on the generic bifurcations in the classification of Meyer and Bruno [13-15] (see also [8]). These are the bifurcations that are typically encountered when one has only a single parameter at hand to steer the system through parameter space, or, equivalently, when one investigates the periodic-orbit families in a given autonomous system as a function of energy. In general one assigns a codimension to each type of bifurcation by counting the number of parameters 
to be controlled in order to encounter it in a general setting. (The class of bifurcations of a given codimension is enlarged when symmetries are imposed on the system [16-20].) The generic bifurcations are accordingly the bifurcations of codimension one. In each of these bifurcations there is a central orbit of period $n$, surrounded by one or two satellite orbits of period $\mathrm{nm}$. The cases $m=1,2,3,4$ are the tangent, period-doubling, period-tripling, and period-quadrupling bifurcations, respectively. There are two types of period-quadrupling bifurcations (island chain and touch-and-go), but only one for all other $m$. All period- $m$ bifurcations with $m \geqslant 5$ follow the island chain pattern.

In [7-9] transitional approximations for the collective contributions were derived that are only valid close to the (generic) bifurcation; far away from the bifurcation they give rise to individual contributions with the wrong amplitudes. See [10-12] for uniform approximations that are valid even far away from the bifurcation, where they asymptotically split into individual contributions with the correct amplitudes. Both types of approximations involve integrals with a phase function $\Phi$ and an amplitude function $\Psi$.

It was demonstrated in [21] that bifurcations of codimension two are frequently felt semiclassically and necessitate a collective treatment even when one steers the system through control space with less than two parameters. This implies that collective contributions of this kind will constitute a basic element in a semiclassical trace formula for systems with a mixed phase space. They indeed played an essential role in the semiclassical determination of the quasienergies of the kicked top [22].

This work is devoted to these bifurcations of codimension two in autonomous Hamiltonian systems with two degrees of freedom or time-periodic Hamiltonian systems with one degree of freedom. The bifurcations are classified and their impact on semiclassical periodic-orbit theory is studied in detail. We derive uniform approximations of collective contributions to the semiclassical traces and discuss certain global bifurcations in conjunction with so-called Stokes transition.

Bifurcations of codimension two manifest themselves in one-parameter studies in certain sequences of generic bifurcations. Sadovskií and coworkers [23, 24] found that such sequences can be explained by normal-form theory [25-28], but did not attempt a classification with respect to the codimension. The classical part of the present study is very much inspired by these works. (Bibliographic notes on multiparametric bifurcations can be found in [29].) The derivation of uniform approximations follows the ideas of [10-12].

This paper is organized as follows. In section 2 we present normal forms for the Hamiltonian that describe the bifurcations of codimension two, they are derived in appendix A. The classification is a straightforward extension of the results for codimension one and is simply achieved by including higher-order terms in an expansion in the canonical variables around the bifurcating orbits. We find that they are again organized by the multiplicity $m$ in analogy to the situation for codimension one. The case $m=3$ is an exception, since no bifurcation of codimension two exists. The codimension-three case, already studied in [21], bears the qualitative features of the other scenarios and is discussed instead.

The corresponding sequences of codimension-one bifurcations involve a tangent bifurcation of period $n m$, followed by a period- $m$ bifurcation that involves another orbit of period $n$.

The normal forms and the sequences of codimension-one bifurcations in the neighbourhood of the codimension-two point in control space (technically speaking, the unfolding of the normal forms) are discussed and illustrated in section 3.

With section 4 we turn to aspects within semiclassical periodic-orbit theory and 
present the starting point for the derivation of collective contributions of bifurcating orbits, consisting of a two-dimensional integral over phase space or a Poincaré surface of section that involves the generating function $\hat{S}$ of the classical stroboscopic map.

The derivation of transitional expressions is a simple task in view of the works of Ozorio de Almeida and Hannay [7, 8]. The uniform expressions, however, require more effort. We present normal forms of the phase function $\Phi$ and the amplitude function $\Psi$.

The details of the derivation are given in appendix B. Specifically, investigating the influence from higher-order terms in the phase function $\Phi$ equips us with a sufficient number of coefficients to guarantee the right stationary-phase limit of the expressions, which are therefore truly uniform. This technique was developed for codimension one by Sieber [10] and subsequently used by Sieber and the present author in [11,12]. While the transitional and the uniform expressions only differ in the amplitude function for codimension one, it turns out that the phase function is also sometimes modified in the present situation. (The cases $m=1,2$ lead to standard diffraction integrals connected to the cusp and butterfly catastrophes, respectively. Among the large number of applications, the transitional approximations have been investigated in connection to bifurcations of closed orbits in [30]. Uniform approximations have not been derived there, however, and the canonically invariant determination of coefficients as well as Stokes transitions are also not discussed.)

In section 5 we discuss certain global bifurcations that become important in the ensuing diffraction catastrophe integrals. They give rise to Stokes transitions in which the contribution of a ghost satellite is switched on or off. The ghosts and transitions arise when the integrals are analysed using the method of steepest descent [31-33]. Stokes transitions have been investigated in the context of diffraction integrals and asymptotic expansions before. A uniform approximation for an isolated transition is given in [34] and has been applied for perturbed cat maps in [35], which is the only treatment of this phenomenon in semiclassics that we know of. The Stokes transitions investigated there, however, occur far away from any other bifurcation and can be regarded as isolated. A transition requires special treatment when it occurs in the immediate neighbourhood of a usual 'local' bifurcation, a situation that is often encountered in mixed systems. The uniform approximations and normal forms derived here can also be employed to describe the Stokes transition of a period- $\mathrm{nm}$ ghost prior to a tangent bifurcation when the so-called 'dominant' orbit involved is real and of period $n$. The complete sequence of local and global bifurcations that we can handle consists of the period- $m$ bifurcation at the central orbit and tangent bifurcations of satellites, followed by Stokes transitions in which ghost satellites once more interact with the central orbit.

We conclude and point out open questions in section 6.

\section{Normal forms of the Hamiltonian for bifurcations of codimension two}

\subsection{Objective}

The local bifurcations to be discussed are instances in which periodic orbits coalesce as parameters are varied. The types of bifurcations generically encountered in a given class of systems depends on the number of parameters varied, and the number of parameters typically needed to be controlled in order to find a particular type is called its codimension. Here we investigate bifurcations of codimension two in the class of periodically time-dependent Hamiltonian systems with one degree of freedom. In other words, we study families of Hamiltonians

$$
H(q, p, t ; \varepsilon, a)
$$


that depend on the two parameters $\varepsilon$ and $a$ and obey $H(t)=H(t+1)$, where the period is set to unity for convenience. In general these systems have no time-reversal nor any geometric symmetry. The discussion directly carries over to autonomous systems with two degrees of freedom since these can be reduced to one-parameter families of periodic systems with one degree of freedom by a standard procedure described for example in [8].

\subsection{The bifurcation condition}

The periodic orbits are seen as fixed points in iterations of the so-called stroboscopic map $(q, p) \rightarrow\left(q^{\prime}, p^{\prime}\right)$ which is induced by the evolution over one period. This map is area preserving. Its linearized version

$$
M=\left(\begin{array}{ll}
\left.\frac{\partial q^{\prime}}{\partial q}\right|_{p} & \left.\frac{\partial q^{\prime}}{\partial p}\right|_{q} \\
\left.\frac{\partial p^{\prime}}{\partial q}\right|_{p} & \left.\frac{\partial p^{\prime}}{\partial p}\right|_{q}
\end{array}\right)
$$

(corresponding to a $2 \times 2$ matrix) hence obeys $\operatorname{det} M=1$. Orbits that appear for the first time in the $n_{0}$ th iteration are said to be of primitive period $n_{0}$. Such an orbit gives rise to $n_{0}$ fixed points in each $n$-step map with $n=r n_{0}$, where $r$ is an integer counting repetitions.

The eigenvalues $\lambda_{1,2}$ of the linearized $n_{0}$-step map $M^{\left(n_{0}\right)}$ are reciprocal to each other. A stable orbit has unimodular eigenvalues and hence $\operatorname{tr} M^{\left(n_{0}\right)}=2 \cos \omega$ with the real stability angle $\omega$. An orbit is instable if the eigenvalues are real. There are two cases depending on the sign of the eigenvalues, $\operatorname{tr} M^{\left(n_{0}\right)}= \pm 2 \cosh \omega^{\prime}$, with the real and by convention positive instability exponent $\omega^{\prime}$.

In general, an orbit bifurcates whenever the linearized $n$-step map $M^{(n)}$ (with again $\left.n=r n_{0}\right)$ acts at the locus of the orbit in phase space in at least one direction as the identity map and hence obeys

$$
\operatorname{tr} M^{\left(r n_{0}\right)}=\operatorname{tr}\left(M^{\left(n_{0}\right)}\right)^{r}=2
$$

or, equivalently,

$$
\operatorname{tr} M^{n_{0}}=2 \cos (2 \pi l / m)
$$

where the integers $l, m$ are taken as relatively prime. This bifurcation condition implies a discrete $m$-fold rotational symmetry $C_{m}$ in the flow pattern around the bifurcating orbit. For $m \geqslant 2$ the orbit in question is a 'central' orbit on which 'satellite' orbits of primitive period $n_{0} m$ contract at the bifurcation. For $m=1$ there are two possibilities, the orbit is either involved as a satellite in a bifurcation with an orbit of smaller primitive period, or it takes part in an isochronous bifurcation with other orbits of same period. Turning these observations around, there is always a central periodic orbit of smallest primitive period $n_{0}$ among the bifurcating orbits which coalesces with satellites of period $n_{0} m$. For that reason $m$ is called the multiplicity.

The bifurcations of codimension one have been classified by Meyer and Bruno [13-15] (see section 1). They constitute the building blocks of the scenarios of higher codimension and will be illustrated together with those of codimension two in the next section. Recall that for each $m$ there is exactly one type with the exception of $m=4$ which allows for two variants.

\subsection{Classification of normal forms}

The bifurcation condition (4) is reflected by the Hamiltonian flow around the central orbit; accordingly, the bifurcations can be investigated by studying the general form of the 
Hamiltonian in the vicinity of this orbit. Following [7, 8, 23, 24, 36], we aim at the reduction of the general expressions to certain simple normal forms by suitable canonical transformations. In that way one can identify the parameters that govern the distance to the bifurcation. They can be chosen such that $\varepsilon=0$ brings us on a codimension-one bifurcation and the codimension-two scenario is encountered if in addition $a=0$.

For codimension one the construction (that is carried out for that case in detail e.g. in [8] and is recapitulated in appendix A) leads to the Birkhoff normal forms

\begin{tabular}{|c|l|}
\hline$m$ & $h^{(m)}(q, p)-H_{0}$ \\
\hline 1 & $\varepsilon q+a q^{3}+\frac{\sigma}{2} p^{2}$ \\
2 & $\varepsilon q^{2}+a q^{4}+\frac{\sigma}{2} p^{2}$ \\
3 & $\varepsilon I+a I^{3 / 2} \cos 3 \phi+b I^{2}$ \\
4 & $\varepsilon I+a I^{2}(1+\cos 4 \phi)+b I^{2}(1-\cos 4 \phi)$ \\
$\geqslant 5$ & $\varepsilon I+a I^{2}+\sum_{l=3}^{[m / 2]} b_{l} I^{l}+c I^{m / 2} \cos m \phi$ \\
\hline
\end{tabular}

For $m \geqslant 3$ they have been expressed in canonical polar coordinates $I, \phi$ with

$$
q=\sqrt{2 I} \sin \phi \quad p=\sqrt{2 I} \cos \phi .
$$

The quantity $H_{0}$ is a constant.

These expressions are autonomous and display the $m$-fold symmetry even globally. The periodic orbits are mapped onto fixed points $\partial H / \partial q=0, \partial H / \partial p=0$ and are thus determined as roots of polynomials in $p$ and $q$. From Vieta's relations between these roots (or locations of satellites) and the coefficients of the polynomials it follows that orbits collapse on the centre as the lowest-order terms $\sim \varepsilon$ (and $\sim a$ for codimension two) of the fixed-point equations are steered to zero.

To describe the codimension-two variants we simply have to include higher-order terms into the normal forms to account for additional satellites that approach the centre. This is carried out in appendix $\mathrm{A}$ and leads to the extended normal forms

\begin{tabular}{|c|l|}
\hline$m$ & $H^{(m)}(q, p)-H_{0}$ \\
\hline 1 & $\varepsilon q+a q^{3}+b q^{4}+\frac{\sigma}{2} p^{2}$ \\
2 & $\varepsilon q^{2}+a q^{4}+b q^{6}+\frac{\sigma}{2} p^{2}$ \\
3 & $\varepsilon I+a I^{3 / 2} \cos 3 \phi+b I^{2}$ \\
4 & $\varepsilon I+a I^{2}(1+\cos 4 \phi)+b I^{2}(1-\cos 4 \phi)+c I^{3}(1+\cos 4 \phi)$ \\
5 & $\varepsilon I+a I^{2}+b I^{5 / 2} \cos 5 \phi$ \\
6 & $\varepsilon I+a I^{2}+b I^{3}+c I^{3} \cos 6 \phi$ \\
$\geqslant 7$ & $\varepsilon I+a I^{2}+\sum_{l=3}^{[m / 2]} b_{l} I^{l}+c I^{m / 2} \cos m \phi$ \\
\hline
\end{tabular}

The normal forms for $m \geqslant 5$ are the usual Birkhoff normal forms; they will, however, be investigated not only for small $\varepsilon$ but also for small $a$. The expressions for $m \leqslant 4$ go exactly one order beyond the Birkhoff normal forms. The expression for $m=3$ actually describes a scenario of codimension three, since two parameters have to be controlled in order to achieve $a=0$ (see section A.4).

\section{Local bifurcation scenarios}

We now discuss in detail the bifurcations of codimension two that are described by the normal forms given in the preceding section. In each case the location of the periodic points, given as the solutions of the fixed-point equations

$$
\frac{\partial H}{\partial q}=0 \quad \frac{\partial H}{\partial p}=0
$$


are investigated as the parameters are varied. Sequences of codimension-one bifurcations are encountered if only one parameter is varied close to a codimension-two point [23, 24]. In principle, all coefficients of the normal form vary with this parameter. Since we are only interested in the qualitative features it suffices to choose $\varepsilon$ as the parameter, taking fixed values for the others (including $a$, which we take finite). Representative scenarios will be illustrated by contour plots of the normal forms. Unstable orbits appear there as saddles while stable orbits correspond to maxima or minima. In all these sequences there is a period- $m$ bifurcation at $\varepsilon=0$ and a tangent bifurcation of satellites at the parameter combinations

\begin{tabular}{|c|l|}
\hline$m$ & Tangent bifurcation of satellites \\
\hline 1 & $\varepsilon=-\frac{1}{4} a^{3}$ \\
2 & $\varepsilon=\frac{1}{3} \frac{a^{2}}{b}$ \\
3 & $\varepsilon=\frac{9}{32} \frac{a^{2}}{b}$ \\
4 & $\varepsilon=\frac{2}{3} \frac{a^{2}}{c}$ \\
5 & $\varepsilon=-\frac{128}{675} a^{3}$ \\
6 & $\varepsilon=-\frac{1}{3} \frac{a}{2}^{2}$ \\
$\geqslant 7$ & $\varepsilon \approx \frac{1}{3} \frac{a^{2}}{b}$ \\
\hline
\end{tabular}

Incidentally, global bifurcations that are of particular interest in the context of uniform approximations are discussed in section 5 .

\subsection{Tangent bifurcations $(m=1)$}

We begin with the discussion of the individual bifurcation sequences and start with the case $m=1$.

In a tangent bifurcation two orbits of the same primitive period coalesce. On one side of the bifurcation both orbits are ghosts, i.e. complex solutions of the fixed-point equations, and their coordinates and other characteristic quantities are related by complex conjugation. On the other side of the bifurcation both orbits are real, one of them being initially stable and the other unstable. The scenario is described by the normal form $h^{(1)}$ which accounts for two periodic orbits \pm at coordinates $p_{ \pm}=0$ and

$$
q_{ \pm}= \pm \sqrt{-\frac{1}{3} \frac{\varepsilon}{a}} .
$$

One often encounters a third orbit of identical period in close neighbourhood (in phase space) to the bifurcating orbits. This orbit must be taken into account, for instance, to obtain a reasonable semiclassical approximation. One then has to work with the extended normal form $H^{(1)}$. The fixed-point equation $\partial H / \partial q=0$ is a real cubic polynomial in $q$ and has three solutions. The number of real solutions is determined by the sign of the discriminant

$$
D=\left(\frac{1}{8} \frac{\varepsilon}{b}\right)^{2}+\frac{1}{4} \frac{\varepsilon}{b}\left(\frac{1}{4} \frac{a}{b}\right)^{3} .
$$

There are three real solutions for $D<0$ and only one for $D>0$ which is then accompanied by two complex ones. Tangent bifurcations are encountered at $D=0$, that is $\varepsilon=0$ or $\varepsilon=-a^{3} /\left(4 b^{2}\right)$.

A sequence of these two tangent bifurcations is depicted in figure 1. The codimensiontwo bifurcation is obtained when $\varepsilon$ and $a$ pass zero simultaneously. If this is done in such a way that the discriminant changes sign then the number of solutions changes from one 

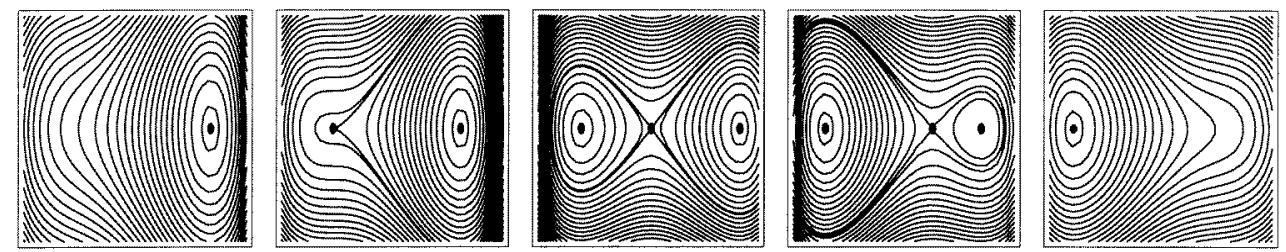

Figure 1. Contour plots of the normal form $H^{(1)}$ as the parameters are steered to cross two tangent bifurcations close to the bifurcation point of codimension two. Initially, only one orbit is present. Two new orbits are born in a tangent bifurcation. One of them approaches the first orbit, and both annihilate in an inverse tangent bifurcation. A similar scenario exists in which stable orbits are unstable and vice versa; it is obtained by reversing the sign of $\sigma$.
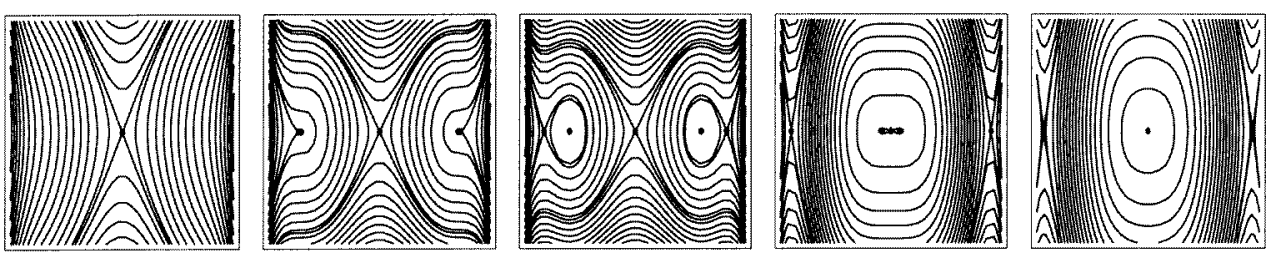

Figure 2. The typical sequence for $m=2$ of a tangent bifurcation of period-two satellites and a period-doubling bifurcation is illustrated by contour plots of $H^{(2)}$. As for $m=1$ there exists a similar scenario for the opposite sign of $\sigma$ in which the stability of orbits is changed. The tangent bifurcation would not be encountered in real phase space if the satellites meet at a negative value of $I$.

to three in a pitchfork bifurcation. Such a bifurcation is even of codimension one if the system is time-reversal symmetric or has a reflection symmetry [16-20].

\subsection{Period-doubling bifurcation $(m=2)$ and tangent bifurcation of satellites}

In a period-doubling bifurcation the central orbit changes its stability by absorbing or emitting a satellite of double period. In the Birkhoff normal form $h^{(2)}$ the central orbit sits at coordinates $q_{0}=p_{0}=0$ and the satellite is represented by two fixed points

$$
p_{1}=0 \quad q_{1}= \pm \sqrt{-\frac{\varepsilon}{2 a}} .
$$

In the extended normal form $H^{(2)}$ the central orbit lies again at $q_{0}=p_{0}=0$, but there are now two satellites \pm with coordinates

$$
q_{ \pm}^{2}=-\frac{1}{3} \frac{a}{b} \pm \sqrt{\frac{1}{9} \frac{a^{2}}{b^{2}}-\frac{1}{3} \frac{\varepsilon}{b}} .
$$

A tangent bifurcation of the satellites is encountered at

$$
\varepsilon=\frac{1}{3} \frac{a^{2}}{b}
$$

but the condition $a b<0$ must be obeyed since otherwise both orbits are still ghosts with purely imaginary $q$-coordinates. A sequence of tangent bifurcation of the satellites and period-doubling bifurcation (with $a b<0$ ) is shown in figure 2 . 

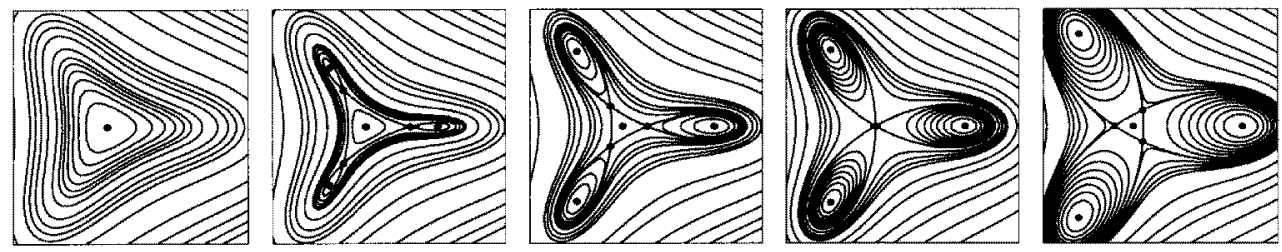

Figure 3. The contour plots of the normal form $H^{(3)}$ display a sequence of a tangent bifurcation of satellites and a period-tripling bifurcation.

\subsection{Period-tripling bifurcation $(m=3)$ and tangent bifurcation of satellites}

The situation for the period tripling is visualized in the sequence of contour plots in figure 3 . Initially, a stable periodic orbit of period one is surrounded by its stability island. At a certain value of the control parameter two satellites of triple period come into existence via a tangent bifurcation. Then the inner (unstable) satellite approaches the central orbit, collides with it in the period tripling, and finally re-emerges on the other side. This scenario has been investigated, for instance, in the diamagnetic Kepler problem [37] and for the kicked top [21].

The Birkhoff normal form $h^{(3)}$ describes the central orbit at $I=0$ and the unstable satellite that is involved in the tripling. The $\phi$-coordinate of the satellite obeys $\partial h^{(3)} / \partial \phi=$ $-3 a I^{3 / 2} \sin 3 \phi=0$. Since a threefold symmetry is implied by this equation it suffices to consider the second equation $\partial h^{(3)} / \partial I=0$ on the $p$-axis after switching back to the coordinates $p, q$, yielding $\varepsilon p+\frac{3}{\sqrt{8}} a p^{2}=0$. Hence the canonical radial coordinate of the satellite is $I=p^{2} / 2=4 \varepsilon^{2} /\left(9 a^{2}\right)$.

In the extended normal form $H^{(3)}$ the $\phi$-coordinates of the satellites again obey $-3 a I^{3 / 2} \sin 3 \phi=0$. On the $p$-axis they now satisfy $\varepsilon p+\frac{3}{\sqrt{8}} a p^{2}+b p^{3}=0$. This equation has three solutions,

$$
p_{0}=0 \quad p_{ \pm}=-\frac{3}{4 \sqrt{2}} \frac{a}{b} \pm \sqrt{\frac{9}{32} \frac{a^{2}}{b^{2}}-\frac{\varepsilon}{b}} .
$$

One in fact sees that the inclusion of the next-order term implies the existence of a further satellite. At $\varepsilon=9 a^{2} /(32 b)$ the satellites undergo a tangent bifurcation and for $\varepsilon / b>9 a^{2} /\left(32 b^{2}\right)$ both satellites are ghosts. For $0<\varepsilon / b<9 a^{2} /\left(32 b^{2}\right)$ both satellites are on the same side of the central orbit, while after the period tripling $(\varepsilon=0)$ they lie opposite to each other. In the limit $\varepsilon / b \rightarrow-\infty$ the satellites form a broken torus, well separated from the central orbit. Three parameters have to be varied in order to achieve $a=\varepsilon=0$, as explained in section A.4. Both satellites are then contracted onto the central orbit in a codimension-three bifurcation.

\subsection{Period-quadrupling bifurcation $(m=4)$ and tangent bifurcations of satellites}

There are two variants of generic period-quadrupling bifurcations depending on the magnitude of the coefficients $a$ and $b$ in the normal form $h^{(4)}$. In both cases there are two satellites of quadruple period involved that lie at $\sin 4 \phi=0$ and are distinguished by the quantity $\cos 4 \phi= \pm 1 \equiv \sigma$. Their radial distance is given by $I^{(\sigma=1)}=-\varepsilon /(4 a)$ and $I^{(\sigma=-1)}=-\varepsilon /(4 b)$. In the touch-and-go case $\operatorname{sign} a=-\operatorname{sign} b$ an unstable satellite becomes a ghost while in turn a ghost solution becomes real and emerges from the central orbit. In the island-chain scenario $\operatorname{sign} a=\operatorname{sign} b$ there are two ghost satellites on one side 

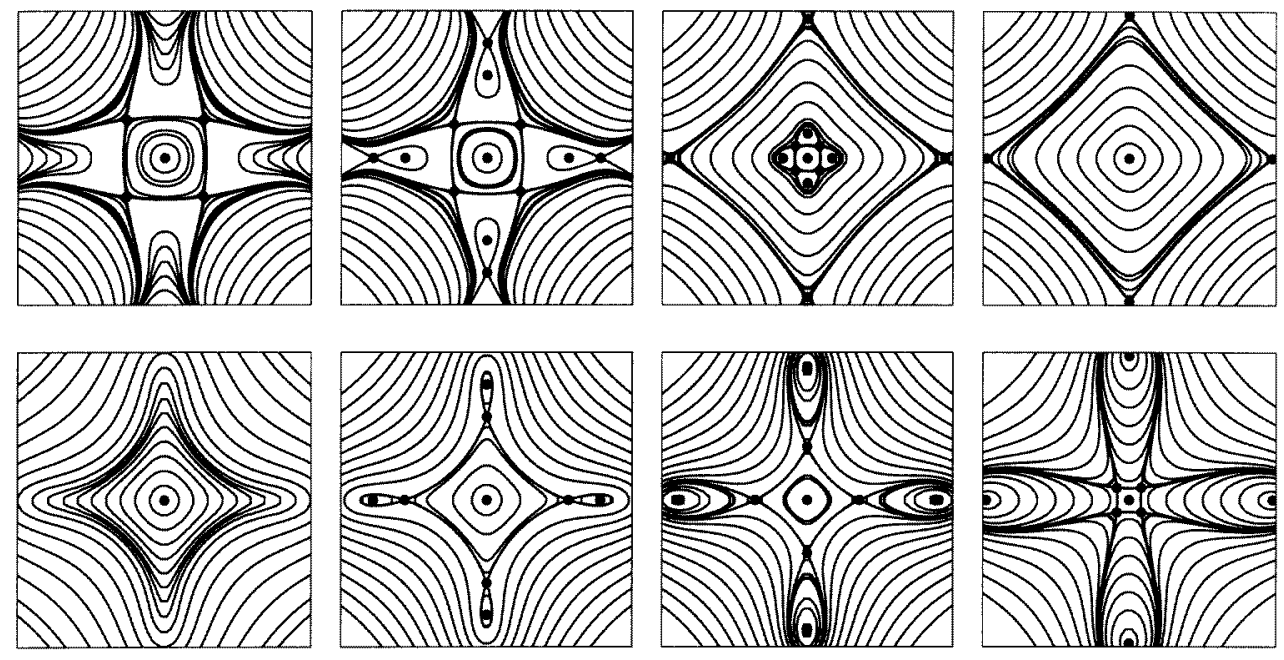

Figure 4. The two sequences of contour plots of $H^{(4)}$ display a tangent bifurcation of satellites followed by a period-quadrupling bifurcation. The latter is encountered in its island-chain version above; below we have the touch-and-go scenario.

of the bifurcation and two real satellites on the other, one of them being stable and the other unstable.

The next-order terms in the extended normal form $\tilde{H}^{(4)}$ equation (A5) involve three new parameters $c, d$ and $e$ and give rise to six satellites. For $e=0$ there are two satellites on the lines $\cos 4 \phi=1$ and two on the lines $\cos 4 \phi=-1$ as well as the two satellites discussed in the derivation of the normal form $H^{(4)}$. There is a tangent bifurcation at $2 a^{2}=3 \varepsilon c$ where the satellites on $\cos 4 \phi=1$ coalesce, and another one at $2 b^{2}=3 \varepsilon d$ that involves the satellites on $\cos 4 \phi=-1$. A great variety of possible configurations of all six satellites exists. Here, however, we are only concerned with the codimension-two bifurcation, described by $H^{(4)}$ and encountered for $\varepsilon=a=0$. It involves only three satellites, those on the lines $\cos 4 \phi=1$ with radial coordinates

$$
I_{ \pm}^{(1)}=-\frac{1}{3} \frac{a}{c} \pm \sqrt{\frac{1}{9} \frac{a^{2}}{c^{2}}-\frac{1}{6} \frac{\varepsilon}{c}}
$$

and that satellite with $\cos 4 \phi=-1$ which is closer to the centre and lies with $H^{(4)}$ at $I^{(-1)}=-\varepsilon /(4 b)$. Compared with the situation described by $\tilde{H}^{(4)}$ the second satellite on the line $\cos 4 \phi=-1$ is shifted to infinity; the two satellites at $I=(b-a) / d$ have now angular coordinates $\cos 4 \phi \approx-5$ and are therefore ghosts. (Certainly they may eventually become real at finite values of $\varepsilon$ and $a$, far away from the codimension-two bifurcation and therefore out of the scope of this work.) A tangent bifurcation is met at $\varepsilon=2 a^{2} /(3 c)$ provided that $I=-a /(3 c)>0$, since the Cartesian coordinates (6) are otherwise imaginary. Sequences of a tangent bifurcation at positive $I$ and the two variants of quadrupling bifurcations are shown in figure 4 .

\subsection{Period- $m$ bifurcation with $m \geqslant 5$ and tangent bifurcations of satellites}

The codimension-one bifurcations for $m \geqslant 5$ follow the island-chain pattern already encountered for $m=4$. There are two satellites that are ghosts on one side of the 

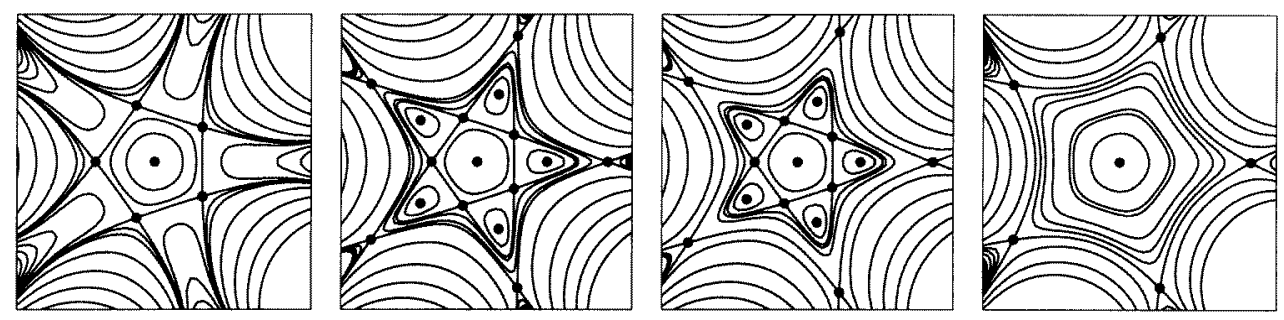

Figure 5. The bifurcation scenario close to the codimension-two point with $m=5$, consisting of a tangent bifurcation of satellites and a period-5 bifurcation.

bifurcation and real on the other, one of them being stable and the other unstable. The stable and unstable periodic points form a chain similar to the broken rational tori that appear in almost integrable systems. Indeed, the $\phi$-dependent terms in the normal forms are of the form of a small perturbation in that situation.

For $m \geqslant 5$ the usual Birkhoff normal forms describe even the codimensiontwo bifurcations: in addition to the orbits participating in the period- $m$ bifurcation of codimension one they also account for the satellites that are involved in the subsequent tangent bifurcations. In the case $m=5$ one obtains three satellites at $\sin 5 \phi=0$ that satisfy on the $p$-axis

$$
\varepsilon+a p^{2}+\frac{5}{4 \sqrt{2}} b p^{3}=0 .
$$

As for $m=1$ it is the discriminant

$$
D=2\left(\frac{2}{5} \frac{\varepsilon}{b}\right)^{2}+\frac{2}{5} \frac{\varepsilon}{b}\left(\frac{8}{15} \frac{a}{b}\right)^{3}
$$

of the equation that governs the number of real solutions. There is the period-5 bifurcation at $\varepsilon=0$ and a tangent bifurcation at $\varepsilon=-128 a^{3} /\left(675 b^{2}\right)$. That sequence is depicted in figure 5 .

In the case $m=6$ one finds four satellites, two on each of the lines $\cos 6 \phi= \pm 1 \equiv \sigma$ at

$$
I_{ \pm}^{(\sigma)}=-\frac{1}{3} \frac{a}{b+\sigma c} \pm \sqrt{\frac{1}{9} \frac{a^{2}}{(b+\sigma c)^{2}}-\frac{1}{3} \frac{\varepsilon}{b+\sigma c} .}
$$

Tangent bifurcations take place at independent parameter combinations $\varepsilon=a^{2} /[3(b+\sigma c)]$ (provided that the $I$-coordinate is not negative). A sequence with two tangent bifurcations at positive values of $I$ is shown in figure 6 . All four satellites approach the centre in the codimension-two bifurcation as $\varepsilon$ and $a$ are sent to zero.

For $m \geqslant 7$ there are even more satellites in the Birkhoff normal form than the four that participate in the codimension-two scenario. In first order, the relevant satellites lie on the lines $\cos m \phi= \pm 1 \equiv \sigma$ at a radial distance

$$
I_{ \pm}^{(\sigma)}=-\frac{1}{3} \frac{a}{b} \pm \sqrt{\frac{1}{9} \frac{a^{2}}{b^{2}}-\frac{1}{3} \frac{\varepsilon}{b}}
$$

which is independent of $\sigma$. The $\phi$-dependent term induces a small correction of order $\sigma I^{m / 2-2}$. Before the tangent bifurcations, which are encountered at almost identical values $\varepsilon \approx a^{2} /(3 b)$, both satellite pairs have complex $I$. After the bifurcation both the inner as well as the outer orbits form island chains that are visible in phase space if the $I$-coordinate is positive. The corresponding sequence is shown in figure 7 . 

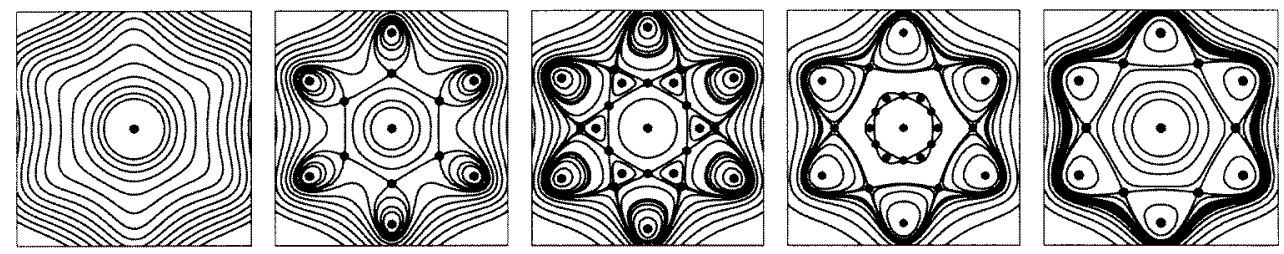

Figure 6. Varying a parameter close to the codimension-two point with $m=6$, one might observe two pairs of satellites being born in a tangent bifurcation at positive $I$, as displayed here; the satellites closer to the centre disappear in a subsequent period- 6 bifurcation. The island chain that is left over here could be also steered to the centre by letting $a$, and then again $\varepsilon$ changes its sign.
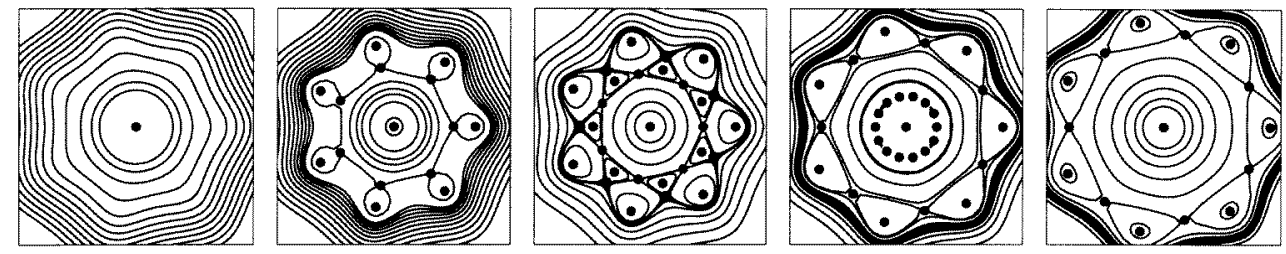

Figure 7. For $m \geqslant 7$ the resonant $\phi$-dependence of the normal form is weak, and tangent bifurcations of satellite pairs occur at almost identical values. Subsequently the inner island chain collapses onto the centre and disappears. The remaining chain might follow, as explained for $m=6$.

\subsection{Absence of alternative bifurcation sequences}

It is an interesting observation that no sequences of period- $m$ bifurcations followed by nontangent bifurcations are encountered in the unfoldings. A prominent example would be taken from a period-doubling cascade, where an orbit of period $n_{0}$ appears as a satellite in a period-doubling bifurcation and has initially $\operatorname{tr} M^{\left(n_{0}\right)}=2$; subsequently it coalesces with a satellite of twice its period at $\operatorname{tr} M^{\left(n_{0}\right)}=-2$. However, we see immediately that both bifurcations cannot be contracted on a single point in control space, since this would lead to a singular change of $\operatorname{tr} M^{\left(n_{0}\right)}$, i.e. the stability properties of the orbit in question.

This reasoning also carries over to other alternative variants of sequences-the bifurcations cannot be contracted in control space on a bifurcation of higher codimensionthis again would entail a singular change in the stability properties of the orbits.

As we explain now in general, only the scenarios with a single central orbit and one kind of satellites are admissible: since we consider here unfoldings, there is a certain point in parameter space where all bifurcations happen simultaneously. This fixes $\operatorname{tr} M^{\left(r n_{0}\right)}$ at the bifurcations in the sequence for each orbit and its repetitions. Specifically, the central orbit of primitive period $n_{0}$ has $\operatorname{tr} M^{\left(n_{0}\right)}=2 \cos (2 \pi l / m)$ with $l$ and $m$ relatively prime and can therefore only coalesce with satellites of period $m n_{0}$. These satellites, on the other hand, have $\operatorname{tr} M^{\left(m n_{0}\right)}=2$ at the period- $m$ bifurcation in the unfolding, which only allows for tangent bifurcations with orbits of identical length as an alternative to the period- $m$ bifurcation with the central orbit.

How would one then deal with cascades? Observe that the various bifurcations will appear at different iterations of the map. We find that the most important scenarios can be studied by iterating the normal-form maps of codimension one, but leave this for future investigations. 


\section{Semiclassical approximations}

We investigate now the impact of bifurcations on semiclassical trace formulae. For autonomous systems with Hamiltonian $H$ we study the trace of the retarded Green function

$$
G(E)=\frac{1}{E+\mathrm{i} 0^{+}-H}
$$

which provides the density of states by

$$
d(E)=-\frac{1}{\pi} \operatorname{Im} \operatorname{tr} G(E) .
$$

Periodically driven systems are described stroboscopically by a time-evolution operator $F$. For convenience we set the stroboscopic period to unity. $F$ represents in general the unitary operator of a quantum map with a classical limit. The eigenstates of $F$ are stroboscopically stationary, and the phases of the unimodular eigenvalues are called quasienergies. The quasienergy spectrum is encoded in the traces $\operatorname{tr} F^{n}$.

It is convenient to consider in the following $\operatorname{tr} F^{n}$ and i $\hbar \operatorname{tr} G(E)$, the factor being introduced to facilitate a parallel investigation of both cases.

\subsection{Periodic orbits in semiclassical approximations}

A semiclassical link between these traces and isolated periodic orbits is provided by Gutzwiller's trace formula [1-4]. The expression is regained from the uniform approximations to be derived in the limit $\hbar \rightarrow 0$ and is therefore of fundamental importance. The traces are given as a sum over the periodic orbits of given energy $E$ or stroboscopic period $n$, where each orbit contributes

$$
C^{(\mathrm{sp})}=T_{0} \frac{\exp \left[\frac{\mathrm{i}}{\hbar} S^{\left(n_{0} r\right)}-\mathrm{i} \frac{\pi}{2} \mu\right]}{\left|2-\operatorname{tr} M^{\left(n_{0} r\right)}\right|^{1 / 2}} .
$$

In the periodically driven case, $T_{0}=n_{0}$ is the primitive stroboscopic period that was introduced in section 2. (Recall that we set the stroboscopic period to unity.) For autonomous systems, the orbits show up as fixed points in the Poincare map on a surface of section. The primitive period $T_{0}$ is then the time $T$ after which one first comes back to the initial point when starting somewhere on the trajectory of the periodic orbit. The orbit shows up as a fixed point in all $n$-step maps on the surface of section where, as before, $n=r n_{0}$, and $r=T / T_{0}$ is an integer counting repetitions.

Besides the period, three canonical invariant characteristic quantities of periodic points enter, the action $S^{(n)}$, the stability factor $\operatorname{tr} M^{(n)}$, and the Maslov index $\mu$. They will also determine the uniform approximations.

The action $S$ is given by the value of

$$
\Phi(q, p)=\hat{S}^{(n)}(q, p)-q p
$$

at the periodic point, where $\hat{S}^{(n)}\left(q_{n}, p_{0}\right)$ is the generating function of the $n$-step map $\left(q_{0}, p_{0}\right) \rightarrow\left(q_{n}, p_{n}\right)$,

$$
\frac{\partial \hat{S}^{(n)}}{\partial q_{n}}=p_{n} \quad \frac{\partial \hat{S}^{(n)}}{\partial p_{0}}=q_{0}
$$

with in addition $\frac{\partial \hat{S}^{(n)}}{\partial E}=T$ in the autonomous case (in which we refer to the $n$th iterate of the Poincaré map). The linearized $n$-step map $M^{(n)}$ was likewise introduced in section 2. 
It is connected to the second derivatives of $\hat{S}^{(n)}$ and involved in the expression through its trace

$$
\operatorname{tr} M^{(n)}=\frac{1+\left(\frac{\partial^{2} \hat{S}^{(n)}}{\partial q_{n} \partial p_{0}}\right)^{2}-\frac{\partial^{2} \hat{S}^{(n)}}{\partial q_{n}^{2}} \frac{\partial^{2} \hat{S}^{(n)}}{\partial p_{0}^{2}}}{\frac{\partial^{2} \hat{S}^{(n)}}{\partial q_{n} \partial p_{0}}} .
$$

Finally, there is the Maslov index $\mu=v-\frac{1}{2} \operatorname{sign} \Phi^{\prime \prime}$ where $v$ is the Morse index and sign $\Phi^{\prime \prime}$ denotes the difference in the number of positive and negative eigenvalues of the matrix

$$
\Phi^{\prime \prime}=\left(\begin{array}{cc}
\frac{\partial^{2} \Phi}{\partial q_{n}^{2}} & \frac{\partial^{2} \Phi}{\partial q_{n} \partial p_{0}} \\
\frac{\partial^{2} \Phi}{\partial q_{n} \partial p_{0}} & \frac{\partial^{2} \Phi}{\partial p_{0}^{2}}
\end{array}\right)
$$

involving second derivatives of $\Phi$.

The isolated contribution (22) may be derived via a stationary-phase approximation from the integral $($ see $[8,10])$

$$
C_{\Omega^{\prime}}=\frac{1}{2 \pi \hbar} \int_{\Omega^{\prime}} \mathrm{d} q^{\prime} \mathrm{d} p \Psi\left(q^{\prime}, p\right) \exp \left[\frac{\mathrm{i}}{\hbar} \Phi\left(q^{\prime}, p\right)-\mathrm{i} \frac{\pi}{2} v\right]
$$

which gives the semiclassical contribution of an arbitrarily chosen region $\Omega^{\prime}$ on the surface of section or phase space. This integral will serve as a starting point for the derivation of collective contributions of bifurcating periodic orbits. The phase function $\Phi$ is connected to the generating function $\hat{S}^{(n)}$ by (23). The amplitude function $\Psi$ reads

$$
\Psi\left(q^{\prime}, p\right)=\frac{1}{n} \frac{\partial \hat{S}^{(n)}}{\partial E}\left|\frac{\partial^{2} \hat{S}^{(n)}}{\partial q^{\prime} \partial p}\right|^{1 / 2}
$$

for the contributions to $\mathrm{i} \hbar \operatorname{tr} G(E)$; for contributions to $\operatorname{tr} F^{n}$ it is given by

$$
\Psi\left(q^{\prime}, p\right)=\left|\frac{\partial^{2} \hat{S}^{(n)}}{\partial q^{\prime} \partial p}\right|^{1 / 2} .
$$

The stationary points at

$$
\frac{\partial \Phi}{\partial q^{\prime}}=0 \quad \frac{\partial \Phi}{\partial p}=0
$$

are precisely the periodic points, and the stationary-phase approximation

$$
C^{(\mathrm{sp})}=\frac{\Psi}{\sqrt{\left|\operatorname{det} \Phi^{\prime \prime}\right|}} \exp \left[\frac{\mathrm{i}}{\hbar} \Phi-\mathrm{i} \frac{\pi}{2}\left(v-\frac{1}{2} \operatorname{sign} \Phi^{\prime \prime}\right)\right]
$$

(with $\Phi^{\prime \prime}$ given in (26)) yields contribution (22) for each periodic orbit.

\subsection{Normal forms for collective semiclassical contributions}

The stationary-phase approximation is based on an expansion of the phase function up to second order around the trajectory,

$$
\Phi\left(q^{\prime}, p\right)=S_{0}-\frac{\omega}{2} q^{\prime 2}-\frac{\sigma}{2} p^{2}+\mathcal{O}(3)
$$

which is, however, only sensible if the stationary points are well separated.

From the bifurcation condition (4) it follows indeed that the individual contribution (22) of an orbit blows up close to a bifurcation and even diverges right at $\operatorname{tr} M=2$. In the twoparameter family of Hamiltonians (1) the stationary-phase approximation is in danger close 
to bifurcations of codimension one and two. In practical applications with small but finite values of $\hbar$, bifurcations are felt in a finite range in parameter space. Even bifurcations of codimension two or higher are typically felt when only a single parameter (frequently, none at all) is varied. This observation (see for example [30, 21, 22]) is indeed our principal incentive.

Collective contributions of orbits which yield regular expressions close to bifurcations of codimension one as a substitute for the stationary-phase result (22) have been investigated in [7-12]. For the derivation of collective contributions of codimension two we proceed analogous to these previous works.

The starting point is provided by the integral (27). Normal forms for the phase function $\Phi$ and the amplitude function $\Psi$ that supersede the quadratic form (32) are then derived by demanding that they lead to uniform collective contributions when inserted into the integral (27). As mentioned in the introduction, besides being regular close to the bifurcation, such approximations split asymptotically into the sum of individual contributions (22) of the bifurcating orbits with the correct phases and amplitudes.

In section 2 we observed that the Hamiltonian normal forms describe in some cases additional orbits that do not participate in the bifurcations of codimension two. In addition they do not provide enough coefficients to yield independent actions and semiclassical amplitudes in the stationary-phase approximation. Sieber [10], partly in collaboration with this author [11, 12], has shown for codimension-one bifurcations how to overcome these restrictions in a systematic way. One has to consider the influence of higher-order terms in the normal forms on the classical properties of the orbits. These terms can be eliminated in the region of almost stationary phase by noncanonical transformations. Such transformations can also be used to get rid of the additional nonbifurcating orbits described by the original normal form. The Jacobian of the transformation enters the amplitude function $\Psi$, which can be simplified further by partial integrations. The procedure is carried out in appendix B. It results in the following normal forms for $\Phi$ and $\Psi$,

\begin{tabular}{|c|l|}
\hline$m$ & $\Phi^{(m)}\left(q^{\prime}, p\right)-S_{0}$ \\
\hline 1 & $-\varepsilon q^{\prime 2}-a q^{\prime 3}-b q^{\prime 4}-\frac{\sigma}{2} p^{2}$ \\
2 & $-\varepsilon q^{\prime 2}-a q^{\prime 4}-b q^{\prime 6}-\frac{\sigma}{2} p^{2}$ \\
3 & $-\varepsilon I-a I^{3 / 2} \cos 3 \phi-b I^{2}$ \\
4 & $-\varepsilon I-a I^{2}(1+\cos 4 \phi)-b I^{2}(1-\cos 4 \phi)-c I^{3}(1+\cos 4 \phi)$ \\
5 & $-\varepsilon I-a I^{2}-b I^{5 / 2} \cos 5 \phi-\varepsilon c I^{3 / 2} \cos 5 \phi$ \\
6 & $-\varepsilon I-a I^{2}-b I^{3}-c I^{3} \cos 6 \phi-\varepsilon d I^{2} \cos 6 \phi$ \\
$\geqslant 7$, odd & $-\varepsilon I-a I^{2}-b I^{3}-I^{3 / 2}(c I+\varepsilon d) \cos m \phi$ \\
$\geqslant 8$, even & $-\varepsilon I-a I^{2}-b I^{3}-I^{2}(c I+\varepsilon d) \cos m \phi$ \\
\hline$m$ & $\Psi^{(m)}\left(q^{\prime}, p\right)$ \\
\hline 1 & $1+\alpha q^{\prime}+\beta q^{\prime 2}$ \\
\hline 2 & $1+\alpha q^{\prime 2}+\beta q^{\prime 4}$ \\
3 & $1+\alpha I+\beta I^{3 / 2} \cos 3 \phi$ \\
4 & $1+\alpha I+\beta I^{2}+\gamma I^{2} \cos 4 \phi+\delta I^{3}$ \\
5 & $1+(\alpha+\beta I) I^{1 / 2} \cos 5 \phi+\gamma I+\delta I^{2}$ \\
6 & $1+(\alpha+\beta I) I \cos 6 \phi+\gamma I+\delta I^{2}+\xi I^{3}$ \\
$\geqslant 7$, odd & $1+(\alpha+\beta I) I^{1 / 2} \cos m \phi+\gamma I+\delta I^{2}+\xi I^{3}$ \\
$\geqslant 8$, even & $1+(\alpha+\beta I) I \cos m \phi+\gamma I+\delta I^{2}+\xi I^{3}$ \\
\hline
\end{tabular}

In $\Phi^{(1,2)}$ we may choose $|\sigma|=1$. The normal forms for $m \geqslant 3$ are expressed in canonical polar coordinates $I, \phi$ defined in equation (6). 
That these normal forms, in contrast to the Hamiltonian ones, indeed provide just the right number of coefficients to account for independent periodic orbits is demonstrated in the following section. Let us already note here that, for instances, a term as $\varepsilon c I^{3 / 2} \cos 5 \phi$ in $\Phi(5)$ for $m=5$ takes care for independent actions of the four bifurcating orbits. Reviewing the derivation in appendix B we see that it cannot be transformed away within the accuracy required of a uniform approximation without introducing other terms (like $I^{3}$ ). That this term scales as $\varepsilon$ provides additional insight in the expected quality of the approximation: if it becomes too large, this hints unduly large influence of higher-order terms, which are in turn related to additional satellite orbits. (More stationary points show up when the degree of the polynomial increases.) It is then likely required to work with normal forms of even higher codimension.

Note that some of the extra terms in the normal forms for $m \geqslant 5$ cannot be expressed 'perturbatively' as $q^{\prime l} p^{k}$ in Cartesian coordinates (6). The substitutions $m \phi=2 \psi$ for $m$ even and $m \phi=\psi$ for $m$ odd provide a potentially useful regularization. The appearance of such terms in the phase function $\Phi$ is in contrast to the situation of codimension one, where the higher-order terms can be transformed away completely.

(Numerically useful expressions for some of the resulting integrals in terms of Taylor series can be found in [30] $(m=1,2)$ and [21] $(m=3)$. The integrals for $m=1,2$ can also be easily evaluated by the method of steepest descent (cf section 5) since they are essentially one-dimensional. For $m \geqslant 3$, however, a two-dimensional steepest-descent manifold might be quite difficult to construct. It is perhaps more convenient to deform only the $I$ coordinate into the complex, yielding a simple steepest-descent contour for each fixed, real $\phi$, and then to perform the $\phi$-integral of finite range.)

\subsection{Determination of coefficients}

In order to obtain uniform collective contributions that split in the stationary-phase limit into a sum of isolated contributions of Gutzwiller type (22) with correct amplitudes and phases, one has to express the coefficients in the normal forms by the classical quantities $S$, $\operatorname{tr} M$ of the orbits. This is achieved by comparing the individual contributions (22) to (31) and described in detail below. Incidentally, also complex solutions of the stationary-phase condition (30) have to be considered in order to obtain enough classical information. These 'ghost orbits' are investigated in detail in section 5. The resulting expressions are then invariant under canonical transformations $[10-12,38,39]$ and are applicable not only in the immediate neighbourhood of the bifurcation, but also far away. (Transitional approximations of the type mentioned in the introduction are obtained if one uses $\Psi=1$ instead, and discards the nonperturbative terms in the phase for $m \geqslant 5$.)

In detail, the properties of the central orbit determine $\varepsilon$ and $S_{0}$, since the stationary point in the origin gives $C^{(\mathrm{sp})}=\exp \left[\mathrm{i} S_{0} / \hbar-\mathrm{i} \frac{\pi}{2}\left(\nu+\frac{1}{2}(\sigma+\operatorname{sign} \varepsilon)\right)\right] / \sqrt{|2 \varepsilon|}$ for $m=1,2$ (recall that $|\sigma|=1)$ and $C^{(\mathrm{sp})}=\exp \left[\mathrm{i} S_{0} / \hbar-\mathrm{i} \frac{\pi}{2}(\nu+\operatorname{sign} \varepsilon)\right] /|\varepsilon|$ for $m \geqslant 3$. The remaining coefficients of the phase function are uniquely determined by the actions of the satellites. It turns out to be helpful to use an ansatz where the coefficients are expressed by scaled positions on a radial line connecting the satellites with the central orbit. For even $m$ with two real satellites on such a line, for instance, we put them on scaled positions at $x_{1}= \pm 1$, $x_{2}= \pm y$, corresponding to

$$
\frac{\mathrm{d} \Phi}{\mathrm{d} x}=A x\left(x^{2}-1\right)\left(x^{2}-y^{2}\right)
$$


integrate to obtain $\Phi(x)$ and determine $y$ from the (scale invariant) ratio

$$
\frac{S_{1}-S_{0}}{S_{2}-S_{0}}=\frac{1-3 y^{2}}{y^{4}\left(y^{2}-3\right)} \text {. }
$$

Without restriction we can demand $0 \leqslant y \leqslant 1$; then there is exactly one solution. The factor $A$ follows from the absolute value of $S_{1}-S_{0}$, and the scale of $x$ is fixed by knowledge of $\varepsilon$. In the case of complex satellites they are placed at $x= \pm 1 \pm$ iy, i.e. $\Phi=A x^{2}\left[x^{4}+3 x^{2}\left(y^{2}-1\right)+3\left(y^{2}+1\right)^{2}\right]$, and $y$ is obtained from

$$
\frac{\operatorname{Re} S_{1}-S_{0}}{\operatorname{Im} S_{1}}=\frac{1+9 y^{2}-9 y^{4}-y^{6}}{16 y^{3}} .
$$

There is a solution with $|y|<1$ and another one with $|y|>1$. The right choice takes into consideration whether the ghost with $\operatorname{Im} S>0$ lies on the steepest-descent contour connecting the integration boundaries or not; see section 5 .

The approach presented here to obtain the coefficients of $\Phi$ works also for the other normal forms. Moreover, the stationary-phase result is a linear combination of the coefficients of the amplitude function $\Psi$, which are therefore easily obtained by comparison with the semiclassical amplitudes in (22). For $m \geqslant 5$ there is a symmetry-related pair of 'spurious' ghost satellites (analogous to those already discussed for $m=4$ ), which are negligible not only since they do not lie on a steepest-descent contour (see again section 5), but also because a 'magic' cancellation in course of the derivation (see section B.7) entails $\Psi=0$ at their positions. Fortunately, exactly one extra term in $\Psi$ shows up in these cases which can be used to achieve this suppression. It seems reasonable that one uses this approach also in the case $m=4$, where an extra coefficient is also at one's disposal.

\section{Stokes transitions}

\subsection{Preliminary remarks}

In the evaluation of the integral representation (27) of contributions to i $\hbar \operatorname{tr} G(E)$ and $\operatorname{tr} F^{n}$, the regions around stationary points come naturally into focus when one uses the steepestdescent method [31-33] to find the leading-order term of an asymptotic expansion of the integral in $\hbar$. To achieve this goal the integration variables are complexified and the initial contour is deformed such that the maxima lie at the solutions of (30), i.e. the periodic points. From the maxima one follows paths of steepest descent of the integrand.

The new contour has to originate from the original one by a continuous deformation without crossing singularities. In order to construct a contour that connects the original integration boundaries one automatically visits also some complex 'ghost' solutions of (30).

The steepest-descent contour constructed so consists of different sheets of constant phase $\operatorname{Re} \Phi$ in the exponent of the integrand, which is given by the real part of the action of the periodic point. For general combinations of the control parameters (codimension zero) there will be only one orbit lying on each sheet, though it is possible that it does so with more than one of the points along its trajectory.

In the stationary-phase limit, only contributions of ghosts that lie on the deformed contour survive. We shall investigate which ghosts do contribute for the collective contributions derived in the preceding section. Specifically, we examine how ghosts become or cease to be relevant as parameters are changed.

To this end, imagine that for one combination of the parameters a ghost lies on the steepest-descent contour while for another one it does not. The ghost is denoted by + in the following and sometimes called 'subdominant' orbit. Its complex conjugate partner is 
denoted by - . Somewhere on a path connecting both parameter combinations the contour changes its form qualitatively in a so-called Stokes transition: The sheet of the ghost merges with the sheet of another orbit, which is called the 'dominant orbit' and denoted by 0 . On both sides of the transition the sheets of the two orbits will connect different zeroes of the integrand. In the transition the contour changes in such a way that the sheet of the subdominant orbit is no longer needed to connect the original integration boundaries.

A necessary condition that the sheets of two orbits merge is that the real part of their actions are identical,

$$
\operatorname{Re} S_{0}=\operatorname{Re} S_{+} .
$$

In general, this condition is not sufficient since both sheets could be separated by others. (Investigating energies of real orbits, the analogous condition $H_{0}=H_{+}$is necessary to find a heteroclinic orbit; again, additional insight is needed to decide whether an equipotential contour joining both orbits indeed exists. These two global bifurcation types are therefore intimately related and constitute, in the language of catastrophe theory, instances of saddle connections.)

\subsection{Stokes transitions in the diffraction integrals}

In the neighbourhood of codimension-two bifurcations one encounters tangent bifurcations in which satellites become ghosts, as was demonstrated in section 3. Subsequently the ghosts may undergo Stokes transitions with the central orbit. We analyse the transitions by investigating the expressions for the actions of the central orbit and the ghosts and using the condition (38).

Besides the tangent bifurcations of the satellites, the unfoldings display also period$m$ bifurcations of codimension one. Let us first demonstrate that no connection can exist between the sheet of the central orbit and ghosts that are born in such a period- $m$ bifurcations of codimension one with $m \geqslant 2$. A helpful rule in that respect is that only ghosts with $\operatorname{Im} \Phi>0$ can lie on the steepest-descent contour. Moreover, satellites that disappear in period- $m$ bifurcations of codimension one with even $m$ are afterwards 'self-conjugated' ghosts, that is, map onto themselves under complex conjugation, and have therefore real classical quantities (in canonical polar coordinates they have real $\phi$ and $I<0$ ); accordingly, they do not contribute. Ghosts immediately beyond a period- $m$ bifurcation of codimension one with odd $m \geqslant 5$ have almost real action, a small imaginary part only being introduced from higher orders, and also do not contribute: in the vicinity of these bifurcations, the ghosts are practically self-conjugated, since the $\phi$-dependent terms in the normal forms of $\Phi$ act only as a small perturbation (see for example, the expressions for the actions in [10]); their presence results only in a slight distortion of the contours, but does not change the situation qualitatively. Since no ghosts are involved in period-tripling bifurcations, we conclude that indeed it suffices to concentrate on ghosts which are born at tangent bifurcations of satellites.

For these ghosts, however, it cannot be avoided to construct the contour in order to find out whether they are relevant or not. (In practical calculations, though, one may be guided by the observation that the majority of relevant ghosts will be close to reality, i.e. about to bifurcate.) Fortunately, it is not difficult to realize that the condition (38) is indeed sufficient for ghosts beyond tangent bifurcations. The reasoning is facilitated by the observation that the problem can be reduced in all cases to one dimension by considering the $I$-lines (or, to be precise, the complex $I$-planes) $\phi \bmod \pi=$ const that connect the central orbit and the ghost satellites radially. 


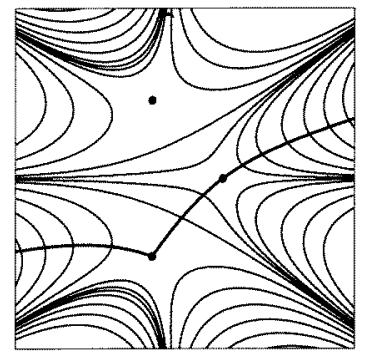

Figure 8. Path of steepest descent $\operatorname{Re} \Phi^{(1)}(q, 0)=S_{0}$ (heavy curve). The parameters are chosen to fulfill the condition for a Stokes transition. The transition indeed takes place since the contour connects the subdominant ghost with the dominant central orbit. The light curves are the equipotential lines of | $\exp [\mathrm{i} \Phi] \mid($ or $\operatorname{Im} \Phi)$.

5.2.1. The cases $m=1, m=3$. For $m=1$ and $m=3$ the situation is simple since there are no other orbits than the two satellites (which are the ghosts in question) and the central orbit. Actually, the functional form of $\Phi^{(3)}$ on the radial line is identical to that of $\Phi^{(1)}$ on the line $p=0$, which allows a parallel treatment. The diffraction integral for $m=1$ involves Pearcey's integral (and its derivatives) for which the Stokes transitions have been studied by Wright [40]. In the construction of $\Phi^{(1)}$ the real solution has been placed in the origin, $q_{0}^{\prime}=p_{0}=0$. According to the stationary-phase condition (30), the satellites have coordinates $p_{ \pm}=0$,

$$
q_{ \pm}^{\prime}=-\frac{3}{8} \frac{a}{b} \pm \sqrt{\frac{9}{64} \frac{a^{2}}{b^{2}}-\frac{1}{2} \frac{\varepsilon}{b}} .
$$

(A tangent bifurcation is now encountered at $\varepsilon=9 a^{2} / 32 b$. Two orbits coalesce also at $\varepsilon=0$, but both remain real there due to the construction.) Inserting the coordinates into the phase function, we obtain that the orbit at the origin has the action $S_{0}$, while

$$
S_{ \pm}=S_{0}-q_{ \pm}^{\prime 2}\left(\frac{\varepsilon}{2}+\frac{a}{4} q_{ \pm}^{\prime}\right)
$$

for the satellites. The Stokes transition takes place at

$$
\varepsilon=\frac{3}{16}(3+\sqrt{3}) \frac{a^{2}}{b} .
$$

Figure 8 displays the integration contour in the complex $q^{\prime}$-plane for $\varepsilon=3(3+\sqrt{3}) / 16$, $a=b=1$ together with the equipotential lines of $|\exp [\mathrm{i} \Phi]|$ (or, equivalently, of $\operatorname{Im} \Phi$ ). The plot demonstrates the well known existence of the connection and is characteristic even for arbitrary sets of parameters that fulfil (41) since the shape of the contour is fully determined by the combination $\varepsilon b / a^{2}$ : The contour expands linearly with a scaling of $q$, such that we can achieve, for instance, $a=b$, and does not change if $\Phi^{(1)}$ is multiplied by a real constant, which allows to set $b=1$.

5.2.2. The case of even $m$. For even $m$, all normal forms are an even polynomial of degree six in the distance $\sim I^{1 / 2}$ on the radial line, and the problem is mapped onto the case $m=2$. We already determined the locations (13) of the orbits for the Hamiltonian normal form $H^{(2)}$. By convention, coefficients changed sign in the definition of $\Phi^{(2)}$, but this does not affect the coordinates of the orbits. The satellites have the actions

$$
S_{ \pm}=S_{0}+\frac{1}{3} \frac{a}{b} \varepsilon-\frac{2}{27} \frac{a^{3}}{b^{2}} \pm 2 b\left(\frac{1}{9} \frac{a^{2}}{b^{2}}-\frac{1}{3} \frac{\varepsilon}{b}\right)^{3 / 2} .
$$

From the condition $\operatorname{Re} S_{ \pm}=S_{0}$ one finds a Stokes transition of complex ghosts for $a=0$ if $\varepsilon b>0$. (No transition is encountered at $\varepsilon=\frac{2}{9} \frac{a^{2}}{b}$ or for $a=0$, but $\varepsilon b<0$, since the radicand is positive then). As for $m=1,3$ there is only one scale-invariant parameter 


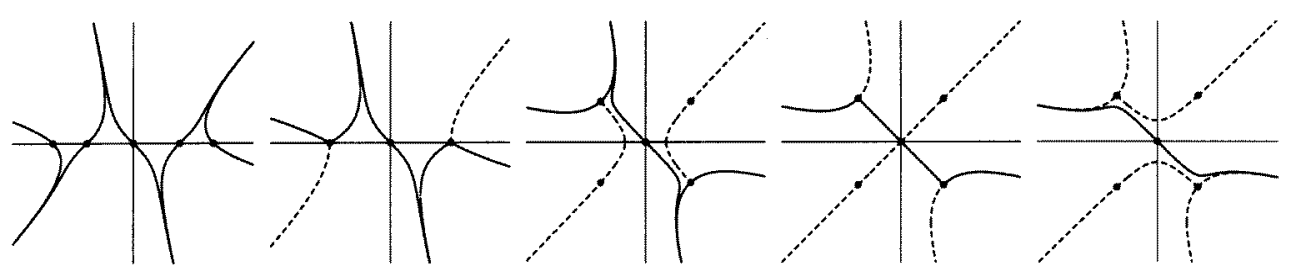

Figure 9. Sequence of steepest-descent contours for $\Phi^{(2)}(q, 0)$ displaying a tangent bifurcation and a Stokes transition. Again there is a connection of the ghost satellite and the central orbit as the condition for a Stokes transition is fulfilled. Dashed lines indicate steepest-descent paths that are not needed to connect the integration boundaries. A trick can be played with these pictures to envision the situation for the tangent bifurcation at negative $I$ : the plots are rotated by 90 degrees (which corresponds to inverting the sign of $a$ ), and the contour is picked that originally connects $\pm \mathrm{i} \infty$ (see also figure 10).

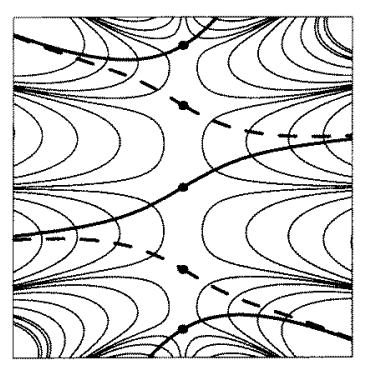

Figure 10. No Stokes transition happens for ghosts with real $I<0$ in the representative case $m=2$. The steepest-descent contours from the ghost and the central orbit (full curves) are separated by the (broken) contour of another ghost at real, but negative $I$. Only the contour that visits the central orbit is needed to connect the integration boundaries.

combination $\varepsilon b / a^{2}$, but for each value there are now two variants of the contour depending on $\operatorname{sign}(a b)$. The Stokes transition at $a=0$ involves a ghost with a nonvanishing imaginary part of the action. Figure 9 shows how the integration contour changes in the complex $q^{\prime}$ plane due to the tangent bifurcation and the Stokes transition. The plots prove the existence of a path that connects both sheets at constant $\operatorname{Re} \Phi$.

Different situations with $\operatorname{Re} S_{ \pm}=S_{0}$ appear, however, for $\varepsilon=\frac{1}{4} \frac{a^{2}}{b}$. No Stokes transition occurs there because the satellite involved is real for $a b<0$ and a ghost with real action and real $I<0$ for $a b>0$. Figure 10 confirms that indeed the contours are separated by another 'real ghost' (broken contour).

5.2.3. The case $m=5$. For $m=5$ the phase function on the $p$-axis is a polynomial of degree five. It initially appears that the three satellites could be arranged in such a way that the sheet of the ghost + is separated from the sheet of the central orbit 0 by the remaining real satellite 1 . One easily finds, however, that the sheets are separated for the particular phase function only if the real parts of the three roots $p_{ \pm}, p_{1}$ of equation (16) all have the same sign, which in turn can be ruled out by a careful inspection of equation (16): from Vieta's relations it follows that otherwise the coefficient of the linear term $\sim\left[p_{+} p_{-}+\left(p_{+}+p_{-}\right) p_{1}\right]$ would not vanish. Note that the order $I^{3 / 2}$ in $\Phi^{(5)}$ indeed results in a nonvanishing coefficient but is considered, as usually, only as a perturbation and does not alter the situation qualitatively. The existence of a steepest-descent connection of the ghost and the central orbit at the Stokes transition is then guaranteed also for $m=5$. The transition takes place at the value of $t \equiv 75 \varepsilon b^{2} /\left(4 a^{3}\right)$ that is the solution of

$$
640+\frac{1520 t}{3}+\frac{925 t^{2}}{9}+t^{3}=0
$$


close to $t=-97.6566$. To derive this equation we first reduce the expression for $\operatorname{Re} S-S_{0}$ with help of the fixed-point equation

$$
8 t+75 r^{2}(4+5 r)=0
$$

for the scaled variable $r \sim p$ to the form $\operatorname{Re}\left[300 r^{2}+t\left(8-10 r+75 r^{2}\right)\right]=0$. Introducing $r=(x+\mathrm{i} y)$ here and in the fixed-point equation and splitting the latter into real and imaginary part, one can solve then for $y^{2}=8 x / 5+3 x^{2}$ and $t=15 x(2+5 x)^{2}$ and obtains the cubic equation $8+60 x+125 x^{2}+75 x^{3}=0$. Its roots give three values for $t$ which all solve (44). Only one of the roots, however, fulfils $y^{2}=8 x / 5+3>0$; this is the one that corresponds to the approximate value of $t$ given above.

5.2.4. The case of odd $m \geqslant 7$. For odd $m \geqslant 7$ there are four different satellites on each line. They are generically grouped in pairs that lie on opposite sides of the centre, and the situation is similar to $m=2$ with slightly broken reflection symmetry. The actions of orbits which lie opposite to each other deviate only due to the influence of the $\phi$-dependent terms in the phase function. These are for $m \geqslant 7$ of higher order than the leading-order terms $-\varepsilon I-a I^{2}-b I^{3}$. The symmetry breaking is therefore neglegible for small $\varepsilon$ (and even for small $a$ ); the results for $m=2$ are then directly applicable to this case.

\section{Conclusions}

We studied bifurcations of codimension two in Hamiltonian systems that are either autonomous and have two degrees of freedom or periodic with one degree of freedom. The normal forms presented in section 2 and discussed in section 3 show that the typical sequence of codimension-one bifurcations in the neighbourhood (in parameter space) of the bifurcation of codimension two consists of a period- $m$ bifurcation at a central orbit followed by a tangent bifurcation in which satellites become ghosts.

Additional generic scenarios are encountered in the presence of symmetries [16-20]. Isochronous pitchfork bifurcations are the most important addition of codimension one in the case of time-reversal or reflection symmetries; they will also show up in the neighbourhood of codimension-two bifurcations in these systems.

Only a small number of the bifurcations of codimension one and two correspond to (special cases of) a so-called elementary catastrophe due to Thom (see e.g. [41, 42]). These appear in many different contexts and describe, for instance, bifurcations of codimension up to four in maps that are not restricted by area preservation. We use the usual names and symbols and further denote each Hamiltonian bifurcation type by $\left(m_{k}\right)$, where $m$ is the multiplicity and $k=1,2$ the codimension. The fold $A_{2}$ corresponds to the tangent bifurcation $\left(1_{1}\right)$. The cusp $A_{3}$ is $\left(1_{2}\right)$, and $\left(2_{1}\right)$ is a cusp with a reflection symmetry. $\left(2_{2}\right)$ is a butterfly $A_{5}$ with reflection symmetry. The period-tripling bifurcation $\left(3_{1}\right)$ corresponds to a version of the elliptic umbilic $D_{4}^{-}$. All other normal forms describe catastrophes that would be of much higher codimension without area preservation. Especially for the cases $m \geqslant 3$ one has to rely on higher-order perturbation theory. It implies that (i) for a given codimension the class of bifurcations in Hamiltonian systems is considerably larger and (ii) although this can be circumvented by considering a normal form of much higher codimension from ordinary catastrophe theory, these normal forms have then again to be restricted: points that correspond to the trajectory of one and the same orbit lie on the same height (energy or action). The classical perturbation theory takes care of this and in addition gives the right codimension. 
Collective contributions to semiclassical traces were derived that involve normal forms for a phase function $\Phi$ and an amplitude function $\Psi$. The expressions involve just as many coefficients as are determined by the actions and stability properties of the bifurcating orbits, including a suppression of certain unwanted ghosts for $m \geqslant 4$. The expressions constitute uniform approximations: they are also valid far away from the bifurcation and asymptotically take the form of a sum of isolated contributions (22).

The validity of the approximations given here is limited if additional orbits become important or 'unwanted' ghosts become real; bifurcations of even higher codimension are then to be studied. The basic steps would be the same as in the present study: derivation of Hamiltonian normal forms that account for all bifurcating orbits; reduction of normal forms to get rid of nonbifurcating orbits and to account for independent stabilities and actions. An important open question is concerned with the complexity of periodic-orbit clusters typically encountered in the quest of resolving spectra when one approaches the semiclassical limit. This requires knowledge of the dynamics up to the Heisenberg time $\sim 1 / \hbar$ and involves a competition of increasing resolution in phase space and proliferation of periodic orbits.

One might also be concerned about cascades, which are sequences of bifurcations of a certain orbit of period $n$ at differing values of $\operatorname{tr} M^{(n)}=2 \cos \omega$ (cf sections 2.2 and 3.6). The most prominent example is the basic building block of period-doubling cascades: an orbit of period $2 n$ is born at an orbit of period $n$ in a period-doubling bifurcation $\left(\operatorname{tr} M^{(2 n)}=2\right)$ and period doubles itself at $\operatorname{tr} M^{(2 n)}=-2$. A huge variety of cascades exists, however, since bifurcations happen whenever the stability angle $\omega$ is a rational multiple of $2 \pi$. We argued in section 3.6 that bifurcations in such a cascade cannot be encountered simultaneously in parameter space, since this would imply a singular change in the linearized map. For that reason cascades cannot be regarded as unfoldings of bifurcations of higher codimension. The bifurcations in an unfolding show up simultaneously in a given iteration of the map; the cascades involve bifurcations that appear in distinct iterations. One could study, for instance, those cascades that arise from the iteration of the map generated by a normal form, and ask the question whether situations exist in which the orbits in the cascade must be treated collectively; it would be indeed nice to see that one can do without. An argument in favour of this expectation has been given in [7].

The uniform approximations derived here display Stokes transitions in which ghost satellites interact once more with the central orbit and leave the steepest-descent integration contour. Stokes transitions are of great practical importance, since they determine the relevance of ghosts in the semiclassical limit. The ghosts which can undergo Stokes transitions originate from the tangent bifurcations of the unfoldings. Ghost satellites are also born in the period- $m$ bifurcations of the unfoldings, but for $m \geqslant 2$ these ghosts do not lie on the steepest-descent contour already directly beyond the bifurcation.

From the experience with the kicked top [22] one can conclude that a reasonable semiclassical quantization scheme for mixed systems has to rely on uniform approximations of codimension two, and that Stokes transitions have to be taken into consideration. It is well possible, however, that much more effort is required when the semiclassical limit is pushed further; periodic-orbit clusters of higher codimension might come into focus as the number of periodic orbits with length up to the relevant time scale (Heisenberg time) increases drastically. 


\section{Acknowledgments}

The author thanks F Haake, C Howls, J Keating, D Sadovskií and M Sieber for helpful discussions. Support by the Sonderforschungsbereich 'Unordnung und große Fluktuationen' of the Deutsche Forschungsgemeinschaft is gratefully acknowledged.

\section{Appendix A. Derivation of normal forms of the Hamiltonian}

We derive here the Hamiltonian normal forms (7) for the codimension-two bifurcations. The procedure is due to Birkhoff [26] and is nicely exposed for example in [8, 24]. In the case of codimension one, it leads to the normal forms (5). In this situation, care has only to be taken in identifying the relevant terms in the expansion for a complete, but simple description of the slightly more complicated bifurcation scenarios in question.

In the derivation of the normal forms (5) and (7) the central orbit is placed into the origin of a local coordinate system $(q, p)$ by a time-dependent canonical transformation. This is done in such a way that a Taylor expansion in $q$ and $p$ yields

$$
H(q, p, t)=H_{0}+\frac{\omega}{2} q^{2}+\frac{\sigma}{2} p^{2}+\mathcal{O}(3)
$$

with time-independent $\omega$ and $\sigma$. The remainder in the expansion indicates third and higher orders in $q$ and $p$. The new Hamiltonian will be $n_{0}$-periodic but otherwise as general as the one we started with. Hence it suffices to study the case $n_{0}=1$.

One passes to a rotating coordinate system, where the angular frequency $2 \pi l / m$ is adopted to the motion around the centre at the bifurcation, and examines the expansion of the Hamiltonian in a Taylor series in $q$ and $p$ as well as a Fourier series in $t$. Most terms in the expansion can be removed by canonical transformations (specified below) up to a certain order in $p, q$ and the deviations of the parameters $\varepsilon, a$ that govern the distance to the bifurcation. The expansion is carried out up to a certain degree, requiring that no bifurcating orbits are added or qualitatively affected by the omitted higher-order terms.

\section{A.1. The cases $m=1,2$}

The linearized map $M$ has degenerate eigenvalues 1 for $m=1$ and -1 for $m=2$. This entails that the corresponding bifurcation scenarios of codimension one and two are essentially one-dimensional. The reason is that the linearized map is in these cases generically not diagonalizable: otherwise one would have located the matrix $M= \pm 1$ in the three-dimensional manifold $\operatorname{Sp}(2, \mathbb{R})$ of real $2 \times 2$ matrices with $\operatorname{det} M=1$ [36]. With only two parameters at one's disposal, however, one generically finds only such matrices with $\operatorname{tr} M= \pm 2$ for which the eigenspace is one-dimensional. The linearized map describes then a shear transformation and acts as the identity in only one direction.

The reasoning can be put onto another footing by resolving the apparent paradox that we can cast the second-order terms into a diagonal form $\omega q^{2} / 2+\sigma p^{2} / 2$ in the immediate neighbourhood of the bifurcation, which involves only two parameters. This diagonalization, however, depends in a singular way on the parameters of the original expansion $a_{1} q^{2}+a_{2} p^{2}+a_{3} q p$. (Note that all three terms are $C_{1}$ - and $C_{2}$-invariant.) Accordingly, three coefficients $a_{i}$ are to be controlled in order to let the second order vanish. Such an argumentation will be used again for $m=4$.

We choose the $q$-axis as the line on which orbits approach each other at the bifurcation. In the $p$-direction it is only the quadratic term which effectively influences the characteristic 
properties of the orbits (energy and stability; subsequently also action). This reduction parallels the famous splitting lemma of catastrophe theory.

With a suitable scaling transformation one can achieve $|\sigma|=1$. The normal form for $m=2$ is symmetric in $q$ since in that case the coordinate system rotates by $\pi$ in each period.

\section{A.2. The cases $m \geqslant 3$}

For $m \geqslant 3$ the central orbit is stable close to the bifurcation so that we can achieve $\sigma=\omega$ in (A1), entailing $\operatorname{tr} M=2 \cos \omega$. It is then convenient to use canonical polar coordinates (6). In the rotating coordinate system the leading-order term in $I$ takes the form $\varepsilon I$ with $\varepsilon=\omega-2 \pi \frac{l}{m}$ in agreement with the bifurcation condition (4). The expansion reads in detail

$H=H_{0}+\varepsilon I+\sum_{k=3}^{\infty} \sum_{l^{\prime}=-\infty}^{\infty} \sum_{m^{\prime}} V_{k l^{\prime} m^{\prime}} I^{k / 2} \cos \left[m^{\prime} \phi+2 \pi\left(m^{\prime} \frac{l}{m}-l^{\prime}\right) t+\phi_{k l m}\right]$

where $m^{\prime}$ runs from $-k,-k+2, \ldots, k$ since only such terms arise from expressions of type $q^{k^{\prime}} p^{k-k^{\prime}}$. Let us assume that all $t$ and $\phi$ dependence is already eliminated up to a certain order $I^{k / 2}$. The majority of terms of this order are then removed by a canonical transformation to new coordinates $J, \theta$ that is generated according to $\frac{\partial G}{\partial I}=J, \frac{\partial G}{\partial \theta}=\phi$, $H^{\prime}=H-\frac{\partial G}{\partial t}$ by the function

$G(\theta, I)=I \theta+I^{k / 2} \sum_{l^{\prime}=-\infty}^{\infty} \sum_{m^{\prime}} G_{k l^{\prime} m^{\prime}} \sin \left[m^{\prime} \theta+2 \pi\left(\frac{m^{\prime} l}{m}-l^{\prime}\right) t+\phi_{k l m}\right]$

with

$$
G_{k l^{\prime} m^{\prime}}=\frac{V_{k l^{\prime} m^{\prime}}}{\omega m^{\prime}-2 \pi l^{\prime}}=\frac{V_{k l^{\prime} m^{\prime}}}{\varepsilon m^{\prime}+2 \pi l m^{\prime} / m-2 \pi l^{\prime}} .
$$

After the transformation we switch back in our notation from $J, \theta$ to $I, \phi$. The coefficient $G_{k l^{\prime} m^{\prime}}$ diverges at the bifurcation if the resonance condition $l^{\prime}=l \frac{m^{\prime}}{m}$ is met. This affects, for instance, all $t$ - and $\phi$-independent terms $\left(l^{\prime}=m^{\prime}=0\right)$. The remaining $\phi$-dependent terms are of type $I^{k / 2} \cos \left(n m \phi-\tilde{\phi}_{k n}\right)$. Here $n=1,2,3, \ldots$ is an integer since $l$ and $m$ are relatively prime, and $k \geqslant n m$ as before. In the orders that appear in the normal forms the latter restriction admits only $n=1$. The $\phi$-dependent term of lowest order in $I$ is generically of type $I^{m / 2} \cos \left(m \phi+\tilde{\phi}_{m 1}\right)$. A shift of $\phi$ eliminates the constant in the cosine. If the coefficient of this term is not small then one can get rid of constants $\tilde{\phi}_{k 1}$ in higher orders $k>m$ by a transformation of the form $\phi=\theta+\sum_{k^{\prime}=1}^{\infty} g_{k^{\prime}} I^{k^{\prime}}$ with suitably chosen coefficients.

\section{A.3. Further reduction for $m=4$}

Additional considerations are needed for $m=4$. The most general expression that goes one order beyond the Birkhoff normal form reads

$$
\begin{aligned}
\tilde{H}^{(4)} H_{0}+\varepsilon I+ & a I^{2}(1+\cos 4 \phi)+b I^{2}(1-\cos 4 \phi) \\
& +(c+d) I^{3}+(c-d) I^{3} \cos 4 \phi+e I^{3} \sin 4 \phi .
\end{aligned}
$$

The codimension-two bifurcation is approached for vanishing $\varepsilon$ and $a$ (or $b$ ), while the other second-order coefficient $b$ (or $a$ ) is finite. Both cases are equivalent and mapped onto each other by a rotation about $\pi / 4$. The normal form $H^{(4)}$ has been written down for small $a$. Since $b$ is finite we can eliminate two of the three third-order terms in (A5) by a canonical 
transformation and achieve $d=e=0$. (The corresponding generating function is of the simple form $G=\theta I-\left(d I^{2} \sin 4 \theta\right) /(8 b)-e I^{2} /(8 b)$ if corrections involving $\varepsilon$ and $a$ are neglected; the complete form is slightly more complicated.)

The fine-print in the derivation for $m=4$ is that two orbits pretend to bifurcate also as we send $a \rightarrow b$. We identify now these orbits and show that for codimension two they are actually ghosts (complex solutions of the fixed-point equations) at a finite distance to the centre. For simplicity we set $e=0$; this does not affect the general line of reasoning. The satellites that concern us solve the fixed-point equation

$$
\frac{\partial \tilde{H}^{(4)}}{\partial \phi}=-4 I^{2}[a-b+(c-d) I] \sin 4 \phi=0
$$

by $I=I^{(0)} \equiv(b-a) /(c-d)$. The other fixed-point equation yields

$$
\cos 4 \phi=-3 \frac{c+d}{c-d}-\varepsilon \frac{c-d}{(a-b)^{2}}+2 \frac{a+b}{a-b} \equiv C^{(0)} .
$$

(These satellites are related by the reflection symmetry $\phi \rightarrow-\phi$ and undergo a pitchfork bifurcation as $\left|C^{(0)}\right|=1$. The symmetry is broken if $e \neq 0$.) For reasons similar to those put forward for $m=1,2$, two parameters have to be controlled in order achieve $I^{(0)}=0$, i.e. $a=b$. For the given parameter combination there is no $\phi$ dependence in the second order of $I$. However, actually there are two independent terms involving $\phi$, namely $\cos 4 \phi$ and $\sin 4 \phi$ - one of them had been eliminated by a diagonalization that is again sensible only if the other has a nonvanishing coefficient. We already used two parameters, then, such that we must assume $\varepsilon$ and $a \approx b$ to be finite. This gives $|\cos 4 \phi| \sim(a-b)^{-2} \gg 1$ and $\cos \phi \sim(a-b)^{-1 / 2}=\mathcal{O}\left(I^{(0)^{-1 / 2}}\right)$, and the Cartesian coordinates (6) indeed remain finite. As announced this shows that the orbits that appeared to bifurcate are complex solutions (with real $p$ and imaginary $q$ ) of the fixed-point equations and stay away from the centre.

\section{A.4. Codimension of the $m=3$ scenario}

We wish to point out here that the bifurcation scenario $a=\varepsilon=0$ for $m=3$ is actually of codimension three. The reasoning is similar to considerations for $m=1,2,4$ in the preceding paragraphs. We argue that actually two external parameters have to be controlled in order to achieve $a=0$. This eliminates simultaneously all $\phi$-dependent terms of order $I^{3 / 2}$, of which two exist, $I^{3 / 2} \sin 3 \phi$ and $I^{3 / 2} \cos 3 \phi$. The bifurcation sequence in the unfolding has nevertheless the qualitative features of the codimension-two cases with $m \neq 3$.

\section{Appendix B. Derivation of normal forms for phase and amplitude}

In section 4.2 we presented normal forms for the phase function $\Phi$ and the amplitude function $\Psi$. In section 4.3 we demonstrated that these normal forms lead to uniform collective contributions of bifurcating orbits when inserted into the integral (27); the coefficients can be determined unambiguously from the classical properties of the orbits. Here we turn to the derivation of the normal forms.

\section{B.1. From Hamiltonian normal forms to the phase function}

First we have to establish the connection between the Hamiltonian normal forms and the phase function $\Phi$, since the expansion in canonical polar coordinates $I, \phi$ is originally carried out in the Hamiltonian. To each normal form of the Hamiltonian there is a corresponding 
expression for the generating function which carries over to the phase function $\Phi$. This function has as many stationary points as the Hamiltonian. The simplest functional form that can be achieved is identical to the normal form of the Hamiltonian, but with altered coefficients. (Observe, however, that although this form of $\Phi\left(q^{\prime}, p\right)$, when expressed by canonical polar coordinates, obeys again rotational symmetries $C_{m}$, this is no longer the case for the map generated by it.) To illustrate this identification we note that the normal forms effectively describe the integrable dynamics of an autonomous system with one degree of freedom. In action-angle variables $J, \psi$ the evolution over a time interval of duration one is generated by

$$
\hat{S}\left(J, \psi^{\prime}\right)=S_{0}+\left(\psi^{\prime}+2 \pi n\right) J-H(J)
$$

such that $J=J^{\prime}$ and $\psi=\psi^{\prime}-\omega(J) \bmod 2 \pi$ with the torus frequency $\omega(J)=\mathrm{d} H / \mathrm{d} J$.

The action variable $J$ is quantized since the angle $\psi$ is only given modulo $2 \pi$; this gives rise to branches of the generating function that are here enumerated by $n$. We circumvent this obstacle by considering the map in Cartesian coordinates, generated by $\hat{S}\left(q^{\prime}, p\right)$, and appealing to the canonical invariance of the leading-order term in the $\hbar$-expansion that we are looking for. (This does not affect the option to shift finally to canonical polar coordinates in the integral expression (27).) The transformation to these variables yields new coefficients: for instance, while the second derivatives of $H$ around a stable orbit involve the bare stability angle $\omega$, we are led for the generating function to the relation (25) with $\operatorname{tr} M=2 \cos \omega$. The coefficients in $\Phi$ are therefore related, but not identical to those in the normal forms of the Hamiltonian, although we will not reflect this in a change of notation; however, minus signs will be introduced following a convention that is motivated by equation (B1).

\section{B.2. Reduction of extended phase functions to normal form}

Following [10-12] on codimension-one bifurcations, we introduce higher-order terms into the phase function $\Phi$ in order to equip ourselves with enough coefficients such that classical properties of the orbits become independent. These terms are then eliminated in the region of almost stationary phase by noncanonical transformations. Such transformations can also be used to get rid of the additional nonbifurcating orbits described by the original normal form. The Jacobian of the transformation enters the amplitude function $\Psi$, which can be simplified further by partial integrations.

The remainder in the phase and amplitude consists of higher orders in $I$ as well as $a, \varepsilon$. We will invoke Vieta's relations to regard the coefficients as certain orders of the typical distance of the satellites to the central orbit. The coefficient $\varepsilon$ is usually the product of such distances, while $a$ is a sum. We will also use that the original normal forms entail $\Psi=$ constant plus corrections of the form $q^{\prime l} p^{k}$. In most cases the precise form of coefficients in the transformations is of no particular interest and therefore only given where this illustrates the method; the expressions are easily obtained, for instance, with the assistance of symbolic mathematical programmes. Only the feasibility of reduction counts. Finally all coefficients in $\Psi$ and $\Phi$ are not to be determined from explicit expansions of Hamiltonians or generating functions but rather from the actions and stability properties of the orbits as explained in section 4.3.

\section{B.3. Reduction for $m=1$}

For $m=1,2,3$ all the periodic points described by the original normal forms are indeed involved in the bifurcation. For $m=1$ there is at least one real orbit which we place into 
the origin by a shift of $q^{\prime}$. This results in the normal form $\Phi^{(1)}$. The three coefficients $\varepsilon$, $a$, and $b$ as well as the value $S_{0}=\Phi^{(1)}(0,0)$ are fixed by the three actions of the orbits and one of the stability factors. From the normal form follows $\Psi=1$; the other stability factors are therefore not yet independent. At a little distance to the bifurcation, however, higher-order terms in the Hamiltonian or the phase function act as a perturbation, and the implied relations between the classical quantities of the orbits are no longer valid. For $m=1$ we consider terms of type $c q^{\prime 5}$ and $d q^{\prime 6}$, i.e.

$$
\Phi\left(q^{\prime}, p\right)=S_{0}-\varepsilon q^{\prime 2}-a q^{\prime 3}-b q^{\prime 4}-c q^{\prime 5}-d q^{\prime 6}-\frac{\sigma}{2} p^{2} .
$$

Higher-order terms involving $p$ effectively do not alter the final expression and can be discarded. In the region of almost stationary phase the higher-order terms act as a perturbation and can be eliminated by substituting $q^{\prime}=Q+A Q^{2}+B Q^{3}$ with suitably chosen coefficients. (The coefficients can be found in general, but are of no interest here. Simple expressions are obtained if we regard $\varepsilon=\mathcal{O}\left(q^{* 2}\right)$ and $a=\mathcal{O}\left(q^{*}\right)$, where $q^{*}$ is the typical distance between the orbits. These orders are justified by Vieta's relations for the stationary-point equation

$$
\left.\frac{\mathrm{d} \Phi}{\mathrm{d} q^{\prime}}\right|_{p=0}=-q^{\prime}\left(2 \varepsilon+3 a q^{\prime}+4 b q^{\prime 2}\right)+\mathcal{O}\left(q^{\prime 4}\right)=0 .
$$

To the required order, we find then $A=-c /(4 b)-27 a c^{2} /\left(128 b^{3}\right)+3 a d /\left(16 b^{2}\right)$ and $B=7 c^{2} /\left(32 b^{2}\right)-d /(4 b)$. $)$

The Jacobian of the transformation involves, in general, $\mathrm{d} q^{\prime}=\mathrm{d} Q\left(1+2 A Q+3 B Q^{2}\right)$ and gives the normal form $\Psi^{(1)}\left(q^{\prime}, p\right)$ announced above. The two additional coefficients are determined by the remaining stability factors $\operatorname{tr} M$ of the satellites. Corrections to $\Phi$ of even higher order would carry over to higher-order terms in $\Psi$. They involve additional coefficients and on first sight allow for ambiguities, but can be eliminated by successive partial integrations. The term of highest order is written as

$$
Q^{l} \sim Q^{l-3} \frac{\mathrm{d} \Phi^{(1)}}{\mathrm{d} Q}+\text { terms of order } l-1, l-2 .
$$

The partial integration of the first term gives an order $\hbar Q^{l-4}$ and a boundary contribution that vanishes for $l \geqslant 4$. In the course of this procedure the constant 1 and the coefficients $\alpha$ and $\beta$ in $\Psi^{(1)}$ acquire next-to-leading order corrections in $\hbar$ that can be discarded and reflect canonically noninvariant properties of the orbits.

\section{B.4. Reduction for $m=2$}

For $m=2$ the higher-order terms have to obey the reflection symmetry $q^{\prime} \rightarrow-q^{\prime}$ and are on the $q^{\prime}$-axis of the form $d q^{\prime 8}$ and $e q^{\prime 10}$. They are eliminated by $q^{2}=Q^{2}+A Q^{4}+B Q^{6}$. The Jacobian involves only even orders of $Q$. Terms of order $l \geqslant 6$ can be eliminated in $\Psi$ by writing

$$
Q^{l} \sim Q^{l-5} \frac{\mathrm{d} \Phi^{(2)}}{\mathrm{d} Q}+\text { terms of order } l-2, l-4
$$

and performing a partial integration of the first term that yields a term $\sim \hbar Q^{l-6}$. This reduces the amplitude function to its normal form $\Psi^{(2)}$. 


\section{B.5. Reduction for $m=3$}

We use the case $m=3$ to illustrate in detail the reduction procedure in canonical polar coordinates and consider the extended normal form

$\Phi\left(q^{\prime}, p\right)=S_{0}-\varepsilon I-a I^{3 / 2} \cos 3 \phi-b I^{2}-c I^{5 / 2} \cos 3 \phi-d I^{3}-e I^{3} \cos 6 \phi$.

We can safely use $\varepsilon=\mathcal{O}\left(I^{*}\right)$ and $a=\mathcal{O}\left(I^{* 1 / 2}\right)$ as upper bounds in orders of the typical distance $I^{*}$ of the satellites to the centre, which is in turn connected with the size of the region of almost stationary phase. Note that this does not impose a restriction on the relative order of these parameters as long as they are small enough. In order to be consistent, however, we have to assume $\varepsilon / a=\mathcal{O}\left(I^{* 1 / 2}\right)$, since otherwise the order $I^{5 / 2}$ would be dominant over $I^{3 / 2}$, in contradiction to our initial assumption that higher orders in $I$ can be treated as a perturbation. The transformation

$$
\begin{aligned}
I=J+\left(-\frac{c}{2 b}\right. & \left.+\frac{3 a}{64 b^{3}}\left(8 b d+8 b e-9 c^{2}\right)\right) J^{3 / 2} \cos 3 \psi \\
& +\left(\frac{c^{2}}{2 b^{2}}-\frac{d+e}{2 b}\right) J^{2}+\left(\frac{e}{b}-\frac{c^{2}}{8 b^{2}}\right) J^{2} \sin ^{2} 3 \psi \\
\phi=\psi+\frac{e}{2 b} J & \sin 3 \psi \cos 3 \psi \\
& +\left(\frac{c}{4 b}+\frac{27}{128} \frac{a c^{2}}{b^{3}}-\frac{3 a(d+e)}{16 b^{2}}-\frac{\varepsilon c^{2}}{24 a b^{2}}+\frac{\varepsilon e}{3 a b}\right) J^{1 / 2} \sin 3 \psi
\end{aligned}
$$

leads to the reduced normal form

$$
\begin{aligned}
\Phi^{(3)}\left(q^{\prime}, p\right)= & S_{0}-\varepsilon J-\left(a-\frac{\varepsilon c}{2 b}-\frac{27 a c^{2} \varepsilon}{64 b^{3}}+\frac{3 a(d+e) \varepsilon}{8 b^{2}}\right) J^{3 / 2} \cos 3 \phi \\
& -\left(b-\frac{3 a c}{4 b}-\frac{81 a^{2} c^{2}}{128 b^{3}}+\frac{9 a^{2}(d+e)}{16 b^{2}}+\frac{c^{2} \varepsilon}{2 b^{2}}-\frac{(d+e) \varepsilon}{2 b}\right) J^{2}+\mathcal{O}\left(I^{* 7 / 2}\right) .
\end{aligned}
$$

It is good to see that the corrections to the parameters $a$ and $b$ are of higher order.

The Jacobian of the transformation is

$$
\begin{aligned}
\Psi^{\prime}=\frac{\partial I}{\partial J} \frac{\partial \phi}{\partial \psi} & -\frac{\partial I}{\partial \psi} \frac{\partial \phi}{\partial J}=1+\left(\frac{c^{2}}{2 b^{2}}-\frac{d}{b}\right) J \\
& +\frac{8 b e-c^{2}}{16 b^{2}}\left(2 \frac{\varepsilon}{a} J^{1 / 2} \cos 3 \psi+J \cos 6 \psi\right)+\mathcal{O}\left(I^{* 3 / 2}\right) .
\end{aligned}
$$

Interestingly, the order $I^{* 1 / 2}$ is absent here due to a cancellation.

For the uniform approximation we need to consider the integral

$$
I_{1} \equiv \int \mathrm{d} J \mathrm{~d} \psi \Psi^{\prime} \exp \left[\frac{\mathrm{i}}{\hbar} \Phi^{(3)}\right] .
$$

In the following partial integrations it will be consistent to disregard the higher-order corrections to the coefficients $a$ and $b$ in the phase (B9). We can use then

$$
\begin{aligned}
& \frac{\varepsilon}{a} \int_{0}^{\infty} \mathrm{d} J J^{1 / 2} \cos 3 \psi \exp \left[\frac{\mathrm{i}}{\hbar} \Phi^{(3)}\right] \\
& \quad=\frac{1}{a} \int_{0}^{\infty} \mathrm{d} J J^{1 / 2} \cos 3 \psi\left(\frac{\partial \Phi^{(3)}}{\partial J}-\frac{3}{2} a J^{1 / 2} \cos 3 \psi-2 b J\right) \exp \left[\frac{\mathrm{i}}{\hbar} \Phi^{(3)}\right]
\end{aligned}
$$




$$
\begin{aligned}
= & -\frac{1}{a} \frac{\hbar}{\mathrm{i}} \int_{0}^{\infty} \mathrm{d} J \frac{1}{2} J^{-1 / 2} \cos 3 \psi \exp \left[\frac{\mathrm{i}}{\hbar} \Phi^{(3)}\right] \\
& -\int_{0}^{\infty} \mathrm{d} J\left(\frac{3}{2} J \cos ^{2} 3 \psi+2 \frac{b}{a} J^{3 / 2} \cos 3 \psi\right) \exp \left[\frac{\mathrm{i}}{\hbar} \Phi^{(3)}\right]
\end{aligned}
$$

and

$$
\begin{aligned}
-\frac{1}{a} \frac{\hbar}{\mathrm{i}} \int_{0}^{2 \pi} \mathrm{d} \psi & \frac{1}{2} J^{-1 / 2} \cos 3 \psi \exp \left[\frac{\mathrm{i}}{\hbar} \Phi^{(3)}\right] \\
= & -\frac{1}{a} \frac{\hbar}{\mathrm{i}} \int_{0}^{2 \pi} \mathrm{d} \psi \frac{1}{2} J^{-1 / 2} \frac{1}{3} \frac{\mathrm{d} \sin 3 \psi}{\mathrm{d} \psi} \exp \left[\frac{\mathrm{i}}{\hbar} \Phi^{(3)}\right] \\
& =\frac{1}{a} \frac{\hbar}{\mathrm{i}} \int_{0}^{2 \pi} \mathrm{d} \psi \frac{1}{2} J^{-1 / 2} \sin 3 \psi \frac{1}{3} \frac{\mathrm{i}}{\hbar}\left(-3 a J^{3 / 2} \sin 3 \psi\right) \exp \left[\frac{\mathrm{i}}{\hbar} \Phi^{(3)}\right] \\
& =-\int_{0}^{2 \pi} \mathrm{d} \psi \frac{1}{2} J \sin ^{2} 3 \psi \exp \left[\frac{\mathrm{i}}{\hbar} \Phi^{(3)}\right]
\end{aligned}
$$

where in both cases the boundary terms vanish. (The boundary term at $J=\infty$ can be discarded after introducing a small imaginary suppression term as $\mathrm{i}^{+} J^{2}$ into the phase.) As a result we obtain

$$
I_{1}=\int \mathrm{d} J \mathrm{~d} \psi \Psi^{(3)} \exp \left[\frac{\mathrm{i}}{\hbar} \Phi^{(3)}\right]
$$

with the new amplitude function

$\Psi^{(3)}=1+\left(\frac{5 c^{2}}{8 b^{2}}-\frac{d+e}{b}\right) J+\left(\frac{c^{2}}{4 a b}-\frac{2 e}{a}\right) J^{3 / 2} \cos 3 \psi+\mathcal{O}\left(I^{* 3 / 2}\right)$.

Note that one could work with alternative forms like $\Psi^{(3)}=1+\alpha I+\beta I^{2}$; the equivalence to $\Psi^{(3)}$ is again worked out by a partial integration.

\section{B.6. Reduction for $m=4$}

Only three of the six satellites described by $\tilde{H}^{(4)}$ (A5) are involved in the codimension-two bifurcation with $m=4$. The normal form $\Phi^{(4)}$ has been given a deeper foundation in the discussion of the corresponding Hamiltonian $H^{(4)}$ in section A.3, where some (canonical) transformations have been performed. The outer satellite on the lines $\cos 4 \psi=-1$ is shifted to infinity and is infinitely unstable, $|\operatorname{tr} M|=\infty$. The two satellites at radial distance $I=(b-a) / c$ have $\cos 4 \psi \approx-5$. They are consequently ghosts with real actions and do not contribute in the stationary-phase limit (for a deeper foundation see section 5); moreover, they are quite far away from the bifurcating orbits. We assume for that reason that their influence is negligible. This leaves us with a normal form that is again completely determined by the actions and one stability factor. We assume that the three non-bifurcating satellites remain negligible even under the influence of higher-order terms.

The reduction of the extended normal form is then carried out in the same way as for $m=3$. The corresponding amplitude normal form $\Psi^{(4)}$ is irreducible under further partial integrations, but has one more coefficient than needed to account for independent stability factors of the satellites. In analogy to the situation to be discussed for $m \geqslant 5$ we can use this coefficient to yield $\Psi=0$ at the position of the unwanted ghosts in favour of an additional suppression. 


\section{B.7. Reduction for $m=5,6$}

For $m=5$ there are three bifurcating satellites but not enough coefficients in the original normal form to account for independent actions. Observe that a scaling transformation $I=A J$ does not affect the values of the phase function at the stationary points. Only the two combinations $\varepsilon^{2} / a$ and $\varepsilon^{5 / 2} / b$ enter these values which have to match the three actions of the satellites. (The coefficients are determined uniquely if one takes the stability factors into consideration.) Independent actions are admissible after allowing for higher-order terms which are once more removed by a transformation in order to yield no spurious additional stationary points. The next-order term, for instance, conveniently expressed as $+5 b c / 2 I^{3}$, can be eliminated by a transformation

$$
\begin{aligned}
& I=J+c J^{3 / 2} \cos 5 \psi \\
& \phi=\psi-\frac{c}{2} J^{1 / 2} \sin 5 \psi
\end{aligned}
$$

which is similar to the one for $m=3$, but now the order $J^{1 / 2}$ survives in the Jacobian,

$$
\Psi=1-c J^{1 / 2} \cos 5 \psi+\mathcal{O}(J) \text {. }
$$

The transformation gives rise to the term $-\varepsilon c J^{3 / 2} \cos 5 \psi$ in $\Phi$ and provides us with the additional coefficient $c$. (The coefficient $\varepsilon c$ in $\Phi$ can be treated as $\mathcal{O}\left(J^{* 3 / 2}\right)$, where $J^{*}$ gives the order of the distance of the satellites to the central orbit. This order is related to the coefficients $\varepsilon$ and $a$ by application of Vieta's relations to the stationary-point equation $\partial \Phi / \partial I=0$.) The next-order corrections to $\Phi$ give even one coefficient more in $\Psi$ than necessary for independent stability factors of the bifurcating orbits. There is, however, an extra pair of satellites at $I=I^{(0)} \equiv-\varepsilon c / b$ that even approaches the centre as $\varepsilon \rightarrow 0$. The angular coordinate, however, obtains a large imaginary part since it obeys $\left(I^{(0)}\right)^{1 / 2} c \cos 5 \phi=1$, where a term of order $a$ has been dropped. Indeed this yields in leading order (B18) a vanishing $\Psi$ and encourages us to use the extra coefficient to accomplish suppression of the unwanted ghosts.

A similar situation is encountered for $m=6$ : only three independent actions of four bifurcating satellites can be modelled with the original normal form, but higher-order terms give rise to corrections that lead to the given normal forms of phase and amplitude. An extra coefficient is again present to suppress the ghost pair at $I=-\varepsilon d / c$, and the Jacobian of the transformation turns out to be once more in favour of such a strategy.

\section{B.8. Reduction for $m \geqslant 7$}

Enough coefficients for the actions are present in the original normal form of $\Phi$ for $m \geqslant 7$, but the expression yields more stationary points than desired. The $\phi$-dependent terms of highest order in $I$ can be eliminated in favour of terms of lower order by successive substitutions $I=J+A J^{l} \cos m \phi$. This procedure can be carried out in parallel to substitutions $I=J+B J^{l}$ that aim at the elimination of $\phi$-independent terms. The coefficients of the remaining terms reflect the values at the stationary point up to the order that yields them as independent from each other and allows also for independent stability factors through the expression for $\Psi$. In the derivation one encounters again a Jacobian that vanishes at the location of the unwanted ghost pair at $I=-\varepsilon d / c$. We should note that the coefficient $c$ is here of order $a$, or, equivalently, $\varepsilon^{1 / 2}$.

\section{References}

[1] Gutzwiller M C 1971 J. Math. Phys. 12343 
[2] Gutzwiller M C 1990 Chaos in Classical and Quantum Mechanics (New York: Springer)

[3] Tabor M 1983 Physica 6D 195

[4] Junker G and Leschke H 1992 Physica 56D 135

[5] Berry M V and Tabor M 1976 Proc. R. Soc. A 349101

[6] Berry M V and Tabor M 1977 J. Phys. A: Math. Gen. 10371

[7] Ozorio de Almeida A M and Hannay J H 1987 J. Phys. A: Math. Gen. 205873

[8] Ozorio de Almeida A M 1988 Hamiltonian Systems: Chaos and Quantization (Cambridge: Cambridge University Press)

[9] Kuś M, Haake F and Delande D 1993 Phys. Rev. Lett. 712167

[10] Sieber M 1996 J. Phys. A: Math. Gen. 294715

[11] Schomerus H and Sieber M 1997 J. Phys. A: Math. Gen. 304537

[12] Sieber M and Schomerus H 1998 J. Phys. A: Math. Gen. 31165

[13] Meyer K R 1970 Trans. Am. Math. Soc. 14995

[14] Bruno A D 1970 Math. USSR Sbornik 12271

[15] Bruno A D 1972 Inst. Prikl. Mat. Akad. Nauk SSSR Preprint No 18 Moskau (in Russian)

[16] Rimmer R J Memoirs of the AMS vol 272 (Providence, RI: American Mathematical Society)

[17] Golubitsky M and Stewart I 1987 Physica 24D 391

[18] de Aguiar M A M, Malta C P, Baranger M and Davies K T R Ann. Phys., NY 180167

[19] de Aguiar M A M and Malta C P 1988 Physica 30D 413

[20] Ozorio de Almeida A M and de Aguiar M A M 1990 Physica 41D 391

[21] Schomerus H 1997 Europhys. Lett. 38423

[22] Schomerus H and Haake F 1997 Phys. Rev. Lett. 791022 a more detailed exposition is in preparation

[23] Sadovskií D A, Shaw J A and Delos J B 1995 Phys. Rev. Lett. 752120

[24] Sadovskií D A and Delos J B 1996 Phys. Rev. E 542033

[25] Poincaré H 1957 New Methods of Celestial Mechanics vol III (New York: Dover)

[26] Birkhoff G D 1927 Dynamical Systems (New York: American Mathematical Society)

[27] Deprit A 1969 Celest. Mech. 112

[28] Arnol'd V I 1988 Geometrical Methods of the Theory of Ordinary Differential Equations (Series of Comprehensive Studies in Mathematics 250) (New York: Springer)

[29] Meyer K R 1986 Contemp. Math. 56373

[30] Main J and Wunner G 1997 Phys. Rev. A 551743

[31] Copson E T 1965 Asymptotic Expansions (Cambridge: Cambridge University Press)

[32] Dingle R B 1973 Asymptotic Expansions: Their Derivation and Interpretation (London: Academic)

[33] Fedoryuk M V 1989 Asymptotic Methods in Analysis, Analysis I (Encyclopaedia of Mathematical Sciences 13) ed R V Gamkrelidze (Berlin: Springer) pp 33-191

[34] Berry M V 1989 Proc. R. Soc. A 4227

[35] Boasman P A and Keating J P 1995 Proc. R. Soc. A 449629

[36] Meyer K R and Hall G R 1992 Introduction to Hamiltonian Dynamical Systems and the N-body Problem (Applied Mathematical Sciences 90) (New York: Springer)

[37] Mao J M and Delos J B 1992 Phys. Rev. A 451746

[38] Tomsovic S, Grinberg M and Ullmo D 1995 Phys. Rev. Lett. 754346

[39] Ullmo D, Grinberg M and Tomsovic S 1996 Phys. Rev. E 54136

[40] Wright F J 1980 J. Phys. A: Math. Gen. 132913

[41] Poston T and Stewart I N 1978 Catastrophe Theory and its Applications (London: Pitman)

[42] Berry M V and Upstill C 1980 Progress in Optics vol VIII, ed E Wolf (Amsterdam: North-Holland) pp 257346 